\title{
INFLUENCE OF REWARD MOTIVATION ON HUMAN DECLARATIVE MEMORY
}

Ewa A. Miendlarzewska ${ }^{a, b,{ }^{*}}$, Daphne Bavelier ${ }^{d^{\dagger}, e}$, Sophie Schwartz ${ }^{a, b, c^{*}}$

${ }^{a}$ Department of Neuroscience, University of Geneva, Geneva, Switzerland

${ }^{\mathrm{b}}$ Swiss Center for Affective Sciences, University of Geneva, Geneva, Switzerland

${ }^{\mathrm{c}}$ Geneva Neuroscience Center, University of Geneva, Geneva, Switzerland

${ }^{d}$ Psychology Section, FPSE, University of Geneva, Geneva, Switzerland

${ }^{\mathrm{e}}$ Brain \& Cognitive Sciences, University of Rochester

* Corresponding authors

Department of Neuroscience, University of Geneva, Geneva, Switzerland Campus BIOTECH

9, Chemin des Mines • 1202 Geneva $\bullet$ SWITZERLAND

Sophie.schwartz@unige.ch Tel: +41 (0) 223795376 Fax: +41 (0) 223795402

Ewa.miendlarzewska@unige.ch Tel: +41 (0) 223790746

${ }^{\dagger}$ Present address

1

(C) 2015. This manuscript version is made available under the Elsevier user license http://www.elsevier.com/open-access/userlicense/1.0/ 


\begin{abstract}
MIENDLARZEWSKA, E.A, D. Bavelier and S. Schwartz. Influence of reward motivation on human declarative memory. NEUROSCI BIOBEHAV REV 44 XXX-XXX, 2015.

Motivational relevance can prioritize information in memory encoding and consolidation based on reward value. In this review, we pinpoint the possible psychological and neural mechanisms by which reward promotes learning, from guiding attention to enhancing memory consolidation. We then discuss how reward value can spill-over from one conditioned stimulus to a non-conditioned stimulus. Such generalization can occur across perceptually similar items or through more complex relations, such as associative or logical inferences. The initial evidence suggests that the neurotransmitter dopamine does not only boost the formation of declarative memory for rewarded information but may also control the generalization of reward values. In particular, temporally-correlated activity in the hippocampus and in regions of the dopaminergic circuit may mediate value-based decisions and facilitate cross-item integration. Given the importance of generalization in learning, our review points to the need to study not only how reward affects later memory but how learned reward values may generalize to related representations and ultimately alter memory structure.
\end{abstract}

Keywords: reward motivation; dopamine; memory; generalization; learning 


\section{Contents}

Abstract

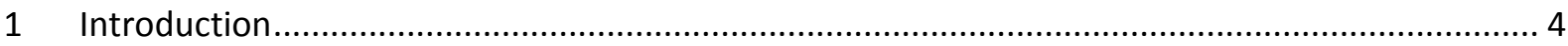

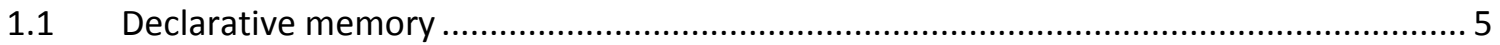

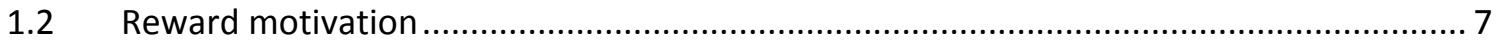

2 Reward motivation promotes learning and memory ....................................................... 7

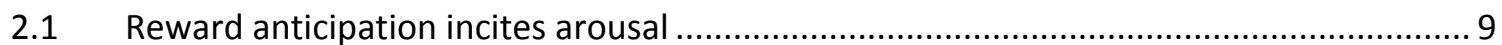

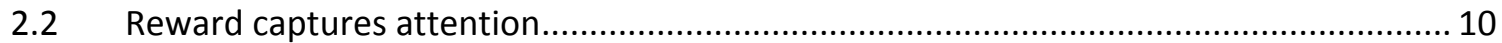

2.3 Role of dopamine in learning and memory consolidation ................................... 12

2.4 Individual differences in reward sensitivity .................................................. 17

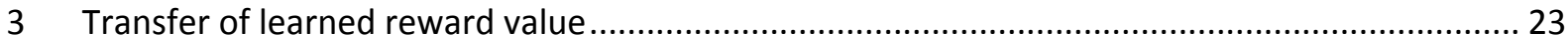

3.1 Reward biases perception and behavior ......................................................... 23

3.1.1 Similarity-based stimulus generalization .................................................. 24

3.1.2 Generalization based on association or acquired equivalence........................... 27

3.1.3 Pavlovian-to-Instrumental Transfer (PIT) ................................................. 29

3.2 When reward motivation enhances declarative memory ..................................... 31

3.2.1 Incidental learning during state of reward motivation ........................................ 31

3.2.2 Reward-related effects on (peri-encoding) memory integration........................... 34

3.2.3 Reward motivation transfer in recollection and memory transformation .............. 36

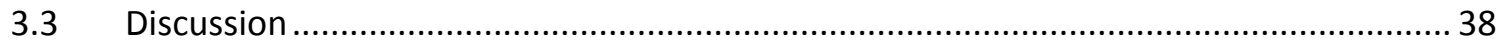

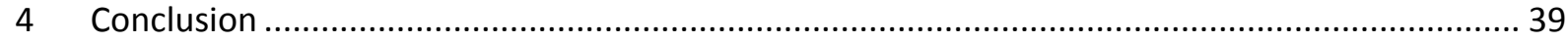

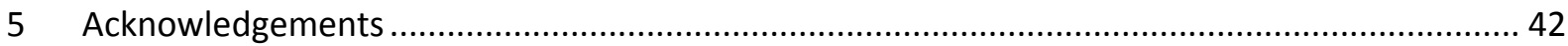

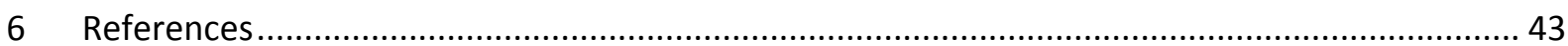

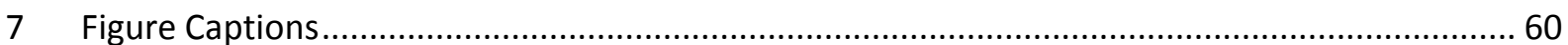

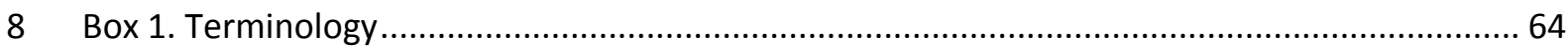

9 Box 2. Role of sleep in the consolidation and generalization of rewarded memories ................66 


\section{Introduction}

Memory is increasingly viewed as an open-ended process that builds on the associative scaffolding of existing knowledge (Wang and Morris, 2010). New memories are often incorporated into existing representations of past experience, so that memory consolidation and retrieval also serve to reactivate and update memory networks (McKenzie and Eichenbaum, 2011; Scimeca and Badre, 2012), some of which may organize into mental schemas (Preston \& Eichenbaum, 2013; see also Box 2). As a result, declarative memory representations may also become more abstract, integrating information across multiple sensory and semantic dimensions.

Through all these processes, memories with an affective relevance are selectively prioritized and better protected from forgetting, compared to emotionally-neutral memories (Mather \& Sutherland, 2011; Talmi, 2013). While a lot is known about the mechanisms promoting aversive (i.e. punishment-predicting) learning, notably thanks to research using variations of the classical fear conditioning paradigm, the impact of rewarding factors on memory processes has received comparatively less attention. However, as we review here, some evidence has recently accumulated to explain how reward may interact with memory formation and underlying neural plasticity.

Below, we provide general definitions for memory systems and reward motivation. Then, we focus on data illustrating that reward modulates attention and memory formation, to a degree dependent on individual traits, such as for example, sensation seeking (section 2). In the second part, we describe research showing that reward value can generalize to new information, and affect learning and memory (section 3). The review thus integrates data from the human behavioral and brain imaging literature to propose a comprehensive view on how reward motivation influences memory, with emphasis on declarative memory. Effects of reward on perceptual (Roelfsema et al., 2010) and motor learning (Wickens et al., 2003) are beyond the scope of the present review, as is memory modulation by emotional relevance, including the effects of fear and fear learning, which have been covered elsewhere (see e.g. Roozendaal and 
McGaugh, 2011). We also discuss findings from animal experiments to better characterize the neural mechanisms underlying the phenomena of memory enhancement and generalization observed in human studies.

To our knowledge, this is the first effort to integrate the far-reaching effects of reward motivation on learning that span phenomena never considered together before, including - among others value generalization through perceptual similarity or various forms of associative learning, as well as the impact of reward motivation transfer in memory recollection and transformation. We end with a brief consideration of potential implications of future research on generalization of rewardlinked memory in the field of education.

[Box 1 about here]

\subsection{Declarative memory}

Declarative memory, further divided into episodic and semantic memory, refers to the capacity to consciously remember past experiences, facts and concepts, and in humans is verifiable through verbal report (Tulving, 1972; see Box 1). It has long been known that the medial temporal lobe (MTL) system (comprising the hippocampus, the dentate gyrus and the subicular complex, together with the entorhinal, perirhinal and parahippocampal cortices; Squire, Wixted, \& Clark, 2007) is necessary for the formation, consolidation, and retrieval of declarative memories (Cohen et al., 1997; Eichenbaum, 2004; Nadel and Moscovitch, 1997; Squire, 1992). Although declarative memory studies often focus on the learning of independent facts, the MTL system is also involved in relational encoding wherein separate episodic elements are bound into an integrated memory trace (Davachi, 2006), and it is also essential for the transfer of learned rules such as making correct inferences for novel combinations of previously learned information (Myers et al., 2003). More and more evidence shows that the hippocampus may not only represent spatiotemporal contexts, but also the relationships between internal motivational states, the external environment, and actions, thus providing a mechanism by which motivation and memory are 
coordinated to guide behavior (Baudonnat et al., 2013; Kennedy and Shapiro, 2009; Wimmer and Shohamy, 2011).

By contrast, other types of non-declarative long-term memory, such as skill learning, habit formation, incremental stimulus-response learning, do not appear to require the MTL system (Corkin, 1968; Knowlton et al., 1994) and have traditionally been associated with the reinforcement learning function of the basal ganglia, and particularly the dorsolateral striatum in rodents which corresponds to the caudate and putamen in humans (Shohamy et al., 2008). Data, however, suggest that these two memory systems may be interacting in either a synergistic or competitive manner (Gheysen et al., 2011; Rose et al., 2011; Sadeh et al., 2011; Schott et al., 2006; Seger et al., 2011), depending on task demands (Delgado and Dickerson, 2012; Packard and Goodman, 2012; Poldrack and Foerde, 2008). Specifically, the declarative memory system involves the MTL cooperating with the ventromedial prefrontal cortex (Preston and Eichenbaum, 2013), and enables instance learning, the extraction and application of underlying associative or categorical rules (generalization) to new instances, as well as retrieval of ensuing episodic and semantic memories (Hoscheidt et al., 2010). The second non-declarative learning system allows for rapid, feedback-based learning of patterns predicting reward, via the recruitment of the basal ganglia (Delgado and Dickerson, 2012; Poldrack and Foerde, 2008), and is essential for habit learning (Liljeholm and O'Doherty, 2012; Schwabe and Wolf, 2013; Smith and Graybiel, 2013). Both systems are capable of some form of associative learning and generalization, and increasing evidence points toward the collaboration between them in post-encoding episodic memory registration (Ben-Yakov and Dudai, 2011) and retrieval (Scimeca and Badre, 2012). Since the exact nature of this interaction is not known, below we consider reward-related modulations of declarative memory and other aspects of behavior separately (section 3).

Although memory formation does not follow a strict step-wise sequence (Nadel et al., 2012), the later description of how reward motivation affects memory refers to distinct processes, as 
proposed by Nadel et al. (2012): (1) encoding, (2) consolidation, (3) recollection (which may lead to reconsolidation, update or disruption of memory), and (4) later transformation.

\subsection{Reward motivation}

Animals and humans are endowed with at least two basic motivational tendencies, namely approach and withdrawal, whose respective activation is associated with positive and negative affective states often referred to as 'rewards' and 'punishments' (Alcaro and Panksepp, 2011). Despite their opposing effects on behavior, the two mechanisms are thought to be mediated by distinct but largely interconnected neural pathways, encompassing limbic and mesolimbic circuits (Alcaro and Panksepp, 2011; Bromberg-Martin et al., 2010b) (see section 2).

Here, we will mainly focus on the approach tendency and define motivation as a state of desire or energy to carry out a certain action, triggered by intrinsic or extrinsic factors (Pennartz et al., 2011). Reward motivation entails the attribution of incentive salience and/or value, which has been summarized by Berridge and Robinson (2003) as cue-triggered 'wanting' (see Box 1).

In the next sections, we report how distinct mechanisms of reward motivation can enhance learning and memory (section 2), and then show how reward motivation can generalize or 'spillover' to later behavior and affect learning (section 3).

\section{Reward motivation promotes learning and memory}

Motivation and memory guide adaptive behaviors. Motivational states direct goals, while memory can inform decision making and actions by recalling information on when and how goals had been obtained or not in the past. Memorizing the pursuit and outcome of motivated actions is therefore important for future choices and underlies optimal adaptation to an ever-changing environment. Reward or motivational relevance can prioritize information in memory based on relative novelty and reward value via dopaminergic projections from the ventral tegmental area (VTA) and the amygdala to the striatum and the hippocampus (Bromberg-Martin et al., 2010b; 
Lisman and Grace, 2005) (see Figure 1). Moreover, motivational salience can be reinstated to some degree every time a previously rewarded memory is retrieved (Luo et al., 2011).

[Figure 1 about here]

In Pavlovian reward conditioning, the value of a reward (for instance, a drop of juice or one dollar) and central and peripheral reactions associated with reward-delivery are transferred to the sensory stimulus (cue) that reliably predicts the reward (Martin-Soelch et al., 2007). Recent evidence from an optogenetic study in rats showed that the mere stimulation of dopaminergic neurons in the VTA/substantia nigra (SN) at the time of reward delivery leads to learning about the predictive value of the preceding cue (Steinberg et al., 2013). Thus, at least in the rat, the firing of dopaminergic neurons is sufficient to produce a teaching signal that results in reinforcement learning. Activity of dopaminergic neurons may actually serve as a critical signal causing goals to become 'wanted' in the sense of motivating actions to achieve them (Berridge, 2012; Bromberg-Martin, Matsumoto, \& Hikosaka, 2010) and driving explorative and approach behaviors, as well as instilling a feeling of anticipatory excitement, or a 'seeking disposition' (Alcaro and Panksepp, 2011).

In more detail, while phasic dopamine increases originating from the VTA indicate cue-triggered response to reward availability (Schultz, 2010, 2007), tonic dopamine levels (minutes to hours), which for example increase during the exploration of novel stimuli, have been suggested to play a role in response vigor (Niv et al., 2007) and overall level of motivation (Ikemoto, 2007; Kaplan and Oudeyer, 2007). Similarly, a delay of reward by several seconds induces a prolongation of both prefrontal dopamine release and reward expectation-related activity in orbitofrontal and striatal neurons (Schultz, 2007), which may be another mechanism for extending the anticipatory excitement in time.

In addition, the interaction between amygdala and the ventral striatum can endow a cue with additional incentive salience (Mahler \& Berridge, 2012; Mahler \& Berridge, 2009). The amygdala 
can selectively increase or decrease the relative value of conditioned stimuli (Bermudez and Schultz, 2010) and provide reward assessment which incorporates reinforcement contingencies (Haber and Knutson, 2010; Morrison and Salzman, 2010; Paton et al., 2006). Below we report how reward motivation, associated arousal and the activation of the mesolimbic dopaminergic system influence attention and memory consolidation. We also make a mention of the possible coinvolvement of noradrenaline and serotonin in reward memory and describe how individual differences influence the response to reward.

\subsection{Reward anticipation incites arousal}

Behavioral arousal, a common feature of positive and negative reinforcement and novelty, is associated with release of noradrenaline (Sara, 2009). In rats, Locus Coeruleus (LC) neurons respond with phasic bursts to both reward and punishment, and to any changes in stimulusreinforcement contingencies (for example, during extinction or reversal) (Sara, 2009). However, while noradrenaline contributes to reward-related invigoration, it is not directly involved in behavioral choice in a reinforcement learning task, unlike the dopaminergic neurons (Glimcher, 2011). Most recently, electrophysiological recordings in behaving monkeys demonstrated that the firing of LC noradrenergic neurons increased with both pupil dilation and effort production in relation to the energization of behavior (Varazzani et al., 2015). At the same time, dopaminergic neurons in the SN pars compacta encoded the expected reward, and their signal decreased in proportion to anticipated effort, which increased the likelihood of the monkey choosing to forgo the trial (Varazzani et al., 2015).

Since noradrenergic and dopaminergic neurons respond to similar environmental stimuli, noradrenaline and dopamine are released simultaneously in some brain areas, such as the frontal cortex, where they act together to modulate network activity (Sara, 2009). In addition, there are reciprocal connections between the two systems and potential release of dopamine from noradrenaline terminals has been suggested (Werlen and Jones, 2015). For example, LC activation 
elicits burst firing in the VTA, resulting in dopamine release in the nucleus accumbens (NAC). LC activation, also via reciprocal projections, affects neurons in the PFC that project indirectly to the VTA. Importantly, the only major region that does not receive input from the LC are the basal ganglia (Sara, 2009), thus precluding a direct role of noradrenaline in motor action selection.

In humans, the effects of arousal on memory are selective and are mediated by $\beta$ adrenoreceptors in the amygdala which purportedly helps detect salient information and recruits the LC when activated (Mather et al., 2015). Like dopamine, noradrenaline modulates memory formation by promoting long-term potentiation (LTP) through these receptors in the amygdala and the hippocampus (Mather et al., 2015; Sara, 2009). Noradrenaline release could thus influence hippocampus-dependent forms of memory during a limited post-learning time window (including slow-wave sleep) (Tully and Bolshakov, 2010). In humans, Sterpenich and colleagues (Sterpenich et al., 2006) showed that emotion-induced changes in pupil response (a marker of LC activity) at encoding correlated with later correct retrieval of emotional stimuli and increased LCamygdala connectivity, thus providing indirect evidence for LC activity enhancing emotionally relevant memories.

\subsection{Reward captures attention}

Reward anticipation boosts incoming sensory signals related to the presented cue, irrespective of whether the perceived stimulus features are task-relevant (Sasaki et al., 2010; Seitz and Watanabe, 2005). Hence, reward affects attention, leading to an increase in correct selection of the reward-associated target amongst other items in the environment and reduced response latency. The recruitment of the dopaminergic reward system is likely responsible for a change in the perceptual representation of stimuli paired with reward value such that these stimuli become salient and attention-drawing (for a review see Gottlieb et al., 2014). Noradrenaline, which enhances signal-to-noise ratio in sensory processing (Sara, 2009), may also be engaged in this process in a state of arousal. Such reward-related attentional effects occur for low-level 
perceptual features, such as color, as well as for more complex stimuli like faces (Raymond and O'Brien, 2009). As a result, perceptual processing of reward-related cues is enhanced, increasing their probability to win the sensory competition for (e.g. visual) cortical representation when presented against a busy background scene (Vuilleumier and Driver, 2007). Moreover, a study in monkeys showed that reward association can continue to affect the value of object representation and gaze direction even months after initial reward learning (Yasuda et al., 2012)., Similarly, a study in humans by Anderson et al. (2011) demonstrated that increased attentional capture by items made salient due to previous reward pairing may persist after a delay of several days. In the experiment, the participants first went through a reinforcement learning phase where they searched for a green or red target among six circles on a display and obtained monetary reward for correctly reporting the orientation of a line segment inside the circle. In a subsequent unrewarded test phase, their task was to search for an oddball square shape in a display of distracter circles, one of which was rendered green or red on half of the trials. The presence of a highly rewarded circular distracter slowed the reaction times when tested immediately as well as at a delay of 4-21 days, and the magnitude of this effect correlated negatively with visual working memory capacity and positively with trait impulsivity. The latter observation suggests that individual differences in reward sensitivity (discussed further in section 2.4 below) affect attentional capture by the reward-signaling stimulus. Faster recognition and behavioral response to reward-predicting cues - in contrast to other cues - is also reflected in the adaptation of the BOLD response. In a neuroimaging study, Zweynert et al. (2011) showed faster suppression of BOLD signal in response to repeated presentations of reward-predictive visual cues in the hippocampus and in ventral visual regions, consistent with a rapid adaptive neural response to rewarded stimuli. Finally, attentional capture by stimuli endowed with incentive salience may contravene the intentional strategy to ignore them in a task where such behavior is actually detrimental to performance because the process is largely automatic (Hickey et al., 2010). Such interference by the salient cue can, for example, lead to slower reaction times (Anderson, 
Laurent, \& Yantis, 2011; Hickey et al., 2010), as well as impaired conflict resolution, demonstrated in the Stroop-type task with rewarded and non-rewarded stimuli (Krebs, Boehler, Egner, Woldorff, \& Carolina, 2011).

\subsection{Role of dopamine in learning and memory consolidation}

Anticipation and consumption of reward influence learning through mechanisms converging in the ventral and dorsal striatum (Pennartz et al., 2011) and involving the release of dopamine by SN neurons. Dopamine neurons are found mainly in the VTA and the neighboring substantia nigra pars compacta (SNpc) (D'Ardenne et al., 2008; Fields et al., 2007; Luo et al., 2011). The dopaminergic signal in the VTA reflects the valence of the feedback received (rewarding or punishing), and the degree to which the feedback was expected, referred to as "prediction error" (Bromberg-Martin et al., 2010a, 2010b; Schultz, 2010). Activity in these dopaminergic neurons signals that a subject's estimate of the value of current and future events is in error and indicates the magnitude of this error (Glimcher, 2011). VTA neurons also project to the hippocampus, where dopamine supports plasticity leading to enhanced memory consolidation (Figure 1, see section 3.2.3 below). Dopaminergic firing often co-occurs with predictive cue presentation, and it has been suggested that it modulates learning by increasing instrumental response vigor (Robbins and Everitt, 2007) or by facilitating the capacity to extract new information from training trials; enhancing memory selectivity and consolidation after learning; and improving performance at a later time (Berridge, 2007).

In their seminal paper, Lisman and Grace (2005) described how dopamine enacts its neuromodulatory role on the hippocampus and how this leads to better episodic memory through a hippocampal-VTA loop (see Figure 1). Dopaminergic neurotransmission from the VTA to the ventral striatum (specifically, the nucleus accumbens) regulates reinforcement learning (Dayan and Balleine, 2002; Fields et al., 2007) but is also important for hippocampal memory consolidation, which begins at the time of encoding. Specifically, dopamine receptor activation in 
hippocampal neurons lowers the threshold for long-term potentiation (LTP) and enhances protein synthesis within the dendrites of these neurons (Smith, Starck, Roberts, \& Schuman, 2005), which is necessary for the maintenance of LTP - the so-called late LTP - in hippocampal area CA1 (Huang and Kandel, 1995). Animal studies suggest that hippocampal memory consolidation may actually critically depend on hippocampal dopamine receptor activation around the time of encoding (Bethus et al., 2010; O'Carroll et al., 2006) as studies on hippocampal place cells indicate their involvement in hippocampal place field formation, stability and remapping to environmental novelty (Werlen and Jones, 2015).

Activating the SN/VTA by reward motivation should therefore enhance hippocampal-dependent episodic memory for information encoded at the time of reward anticipation, and this improvement should be small with short delays when early LTP is present, but comparatively large at longer delays after late LTP has been sustained for selected memory traces (12h after memory acquisition, Caroni et al., 2014; Lisman et al., 2011). Importantly, the memory-stabilizing effect of dopamine extends to other information encoded when dopamine is available, a phenomenon also referred to as the 'penumbra hypothesis' (Lisman et al., 2011). The hypothesis predicts that once dopamine availability is enhanced, any encoded information will benefit from preferential consolidation, regardless of the actual trigger of the dopamine release (Lisman et al., 2011), and initial evidence from rodent (Ballarini et al., 2009; Wang et al., 2010) and human (Fenker et al., 2008; Wittmann et al., 2011) data points out that it may indeed be so. Boosts in dopamine availability at encoding thus result in memory being more stable and better protected from forgetting, an effect that may predominantly arise at long $(>24 \mathrm{~h})$ but not short time intervals after learning, highlighting the role of memory consolidation processes and sleep in dopaminergic modulation of memory traces (See Box 2 on the role of sleep in consolidation; Perogamvros and Schwartz, 2012). 
Note that the concentration of dopaminergic terminals in hippocampus is relatively low when compared to the striatum or PFC (Fields et al., 2007; Werlen and Jones, 2015). Dopamine neurons densely innervate the striatum, the amygdala and the frontal cortex, while according to some anatomical studies in the rat, only $\sim 15 \%$ of VTA and SN neurons that project to the hippocampus are dopaminergic (Fields et al., 2007; Gasbarri et al., 1994). This raises the possibility that hippocampal dopamine may also stem from sources other than the SN, such as the LC itself (Smith and Greene, 2012), because dopaminergic and noradrenergic systems are uniquely intertwined in hippocampal circuits (see section 2.1 above). As a precursor of noradrenaline, dopamine is contained in all noradrenergic fibers and could be released at noradrenergic terminals from the LC which are numerous in the hippocampus (Werlen and Jones, 2015).

Reward related areas are also strongly interconnected with serotonergic neurons. Serotonergic neurons project to neurons in the mesolimbic dopamine system, especially in the NAcc, and regulate dopamine transmission via different serotonin receptor subtypes (Kranz et al., 2010). However, both increasing and decreasing effects of serotonin on reward have been reported (Kranz et al., 2010) and, in contrast to dopamine, serotonin does not appear to be critically involved in hippocampal memory formation (Thomas, 2015; Werlen and Jones, 2015). Several theories proposed that serotonin signals "punishment prediction error" as an opponent to dopamine (Cools et al., 2011; Doya, 2002), however recent evidence does not support this proposal. A study in behaving monkeys (Bromberg-Martin et al., 2010a) showed that in contrast to dopaminergic neurons in the $\mathrm{SN}$, dorsal raphe-nucleus neurons are modulated in a rewarddependent manner before and after reward delivery in a tonic firing pattern related to expected and received reward values. One study in humans showed that serotonin depletion affected the discounting of delayed rewards, whereby small immediate monetary reward was preferred over a larger but delayed one (Schweighofer et al., 2008). Subsequently, it has been suggested that the same serotonin systems are engaged in waiting for rewards as well as in delaying responding to avoid punishment, i.e. passive avoidance (Faulkner and Deakin, 2014). Therefore, serotonin 
released by dorsal raphe nucleus may provide an anticipatory signal for both rewarding and punishing motivational outcomes (Rygula et al., 2014).. At the same time, serotonergic effects on hippocampus are indirect and intricate. Depending on the type of receptor, serotonin agonists may increase or decrease memory performance in laboratory animals and many of these effects also involve the modification of cholinergic, dopaminergic, GABAergic or glutamatergic transmission (Seyedabadi et al., 2014). In human experiments, central serotonin depletion impairs the encoding, delayed but not immediate recall, as well as delayed recall of source episodic memory in a verbal learning task. However, it does not impair spatial memory nor does it affect spatial episodic or general declarative memory (Mendelsohn et al., 2009). In sum, while serotonin does not appear to play a clear role in reward memory formation, and because noradrenaline mainly enhances sensory processing and general invigoration (section 2.1), we consider that the type of reward-induced modulation of declarative memory discussed in the remaining part of the review is primarily caused by dopamine release.

In human fMRI studies, reward delivery and anticipation as well as the co-activation of the VTA have notably been associated with hippocampal memory enhancement, evident upon immediate testing for incidentally learned visual scenes (Wittmann et al., 2005; in purple, Figure 2A) or visual paired associates (Wolosin et al., 2012), as well as in delayed (24h to 3 weeks) recognition memory tests (Adcock, Thangavel, Whitfield-Gabrieli, Knutson, \& Gabrieli, 2006; in green, Figure 2A). Furthermore, data suggest that the interaction of VTA and hippocampus during encoding may contribute to the generalization of item associations (Shohamy \& Wagner, 2008; in light blue, Figure $2 \mathrm{~A}$ ), implying that dopamine also supports the hippocampus in learning predictive rules and developing inference (see section 3.2.2 below). Also consistent with Lisman \& Grace's model (Lisman and Grace, 2005), novelty may trigger dopamine release, which in itself immediately boosts the consolidation of memory traces of novel stimuli (Krebs, Heipertz, Schuetze, \& Duzel, 2011; Lisman et al., 2011; Schott et al., 2004). Novelty may actually interact with reward motivation to additively enhance ventral striatal representation of reward for novel items 
(Guitart-Masip et al., 2010; Krebs, Schott, \& Düzel, 2009; Schott et al., 2004). Response to novel and reward items, compared to rewarded but familiar ones, correlated with an increase in functional connectivity of the medial SN/VTA with mesolimbic regions, including the NAc and the hippocampus (Krebs et al., 2011). A recent study additionally demonstrated that increase of cerebral dopamine by pharmacological manipulation led to reduction in neuronal repetition suppression within the hippocampus, parahippocampal cortex, and substantia nigra/ventral tegmental area, the degree of which was correlated with recognition memory performance $24 \mathrm{~h}$ later (Bunzeck et al., 2014), confirming the dominant role of dopamine in novelty-induced memory enhancement.

[Figure 2 about here]

Although some human studies found effects of reward on immediate memory (Wolosin et al., 2012), there is also evidence that these effects may preferentially emerge after a period of time is allowed for consolidation (Adcock et al., 2006). Using monetary reward cues on one set of line drawings, Wittmann et al. (2005) showed that memory was significantly better for rewarded than non-rewarded drawings when tested three weeks after encoding, but not upon immediate testing. Successful recall of reward-predicting drawings was linked to increased activation of the hippocampus and VTA during initial encoding. A study by Murayama and Kuhbander (2011) also suggested that reward has an enhancing effect on long-term retention but not on immediate declarative memory. The authors used trivia questions the answer to which was either rewarded with a small monetary reward or not. Results of the test conducted 10 min after the quiz showed no difference between the two conditions (possibly due to ceiling effects in recall as the participants achieved $\sim 80 \%$ accuracy rate). One week later, however, average recall of the answers was significantly higher for the initially rewarded answers compared to the nonrewarded condition. These accounts confirm that the effect of reward on declarative memory 
may enhance long-term retention, consistent with the role of dopamine in controlling late-LTP and long-term memory storage (Rossato et al., 2009).

In sum, reward motivation may have distinct effects for short and long-term memory, with more pronounced differences coming into light at longer retention intervals that include off-line replay (Box 2), which favorably protects reward-linked memories from forgetting (Abe et al., 2011).

[Box 2 here]

\subsection{Individual differences in reward sensitivity}

As pointed out by Berridge (2007), individuals may differ in their relative response to the type or amount of reward offered in the experiment. Consequently, also the effects of reward on memory enhancement may depend on its magnitude or selectivity. The response depends on the perceived or anticipated value of the reward given the current state of the organism (e.g. hungry/satiated; 'marginal utility'; Schultz et al., 2008). For instance, the magnitude of the response of the NAc to the delivery of the highest monetary reward in a given task ( 5 dollars) correlated with the individuals' reported happiness during anticipation of the reward (Knutson et al., 2001). Clithero et al. (2011) measured relative individual motivation level for 2 types of rewards as a ratio of reaction time on trials with the preferred reward (e.g. candy bars) relative to the other non-preferred reward (e.g. money). This index was found to be proportional to the BOLD response of the NAc and anterior insula (Clithero et al., 2011) - a region integrating representations of feeling states and uncertainty with individual attitudes (Tobler et al., 2007), such as risk aversion (Singer, Critchley, \& Preuschoff, 2009). Furthermore, not only dopamine signaling of relative incentive salience but also the concurrently formed memory traces are stronger when the reward cue is particularly sought for, as illustrated by the example of human participants who were able to remember images of food with higher accuracy if encoded in hungry as compared to satiated state (Morris and Dolan, 2001). 
This subjective valuation of an extrinsic reward also depends on individual preferences as well as personality traits and underlying genetic profiles (Cohen et al., 2005; Hahn et al., 2009). For example, carriers of genetic polymorphisms of COMT val158met (the enzyme catechol-Omethyltransferase, responsible for the degradation of dopamine in the synaptic cleft), the Met/Met and Val/Val, show different behavior in tasks that rely on dopaminergic transmission. Individuals with the Met allele, characterized by higher availability of prefrontal dopamine, showed significantly higher reward responsiveness in a reward-based probabilistic classification task, as well as higher risk taking in the Balloon analogue risk task ${ }^{\ddagger}$ (Lancaster et al., 2012). This genetic polymorphism was also found to be associated with better suppression of interfering memories, resulting in more efficient remembering (Wimber et al., 2011), pinpointing the role of (prefrontal) dopamine in both reward and memory functions. In that respect, certain aspects of reward-induced approach behavior could be considered as a proxy of dopamine function in memory integration and selective consolidation. However, as we will emphasize in section 3.3, more research is needed to examine the correlation between these two facets of dopamine function.

In rodent research, incentive salience of a Pavlovian cue is a threefold property: the cue is attractive (induces approach behavior), desired (animals work to obtain the cue), and stimulates instrumental actions (animals can learn to perform a new action to get the cued reward; Berridge \& Robinson, 2003; Meyer et al., 2012; Pecina, Cagniard, Berridge, Aldridge, \& Zhuang, 2003). Yet, the attribution of incentive value is not always expressed by the same behavior in all rats. During appetitive conditioning, when the cues signaling the arrival of the food and the location of the food pellet are at different locations, some rats would learn to approach the location of food delivery ('goal-trackers') while others would rather approach the illuminated lever or the

\footnotetext{
${ }^{\ddagger}$ The BART is a risky decision task that involves pumping up a series of virtual balloons in order to earn reward. Balloons may be 'cashed out' to collect earnings at any time, with larger balloons yielding greater earnings. However, each additional pump increases the risk of the balloon explodes which would results in loss of all earnings for that balloon.
} 
conditioned cue (CS+), as if such a sign had higher incentive value than the food itself ('signtrackers', Flagel et al., 2011; Meyer et al., 2012). The latter behavior is a conditioned orienting response and indicates that incentive salience has been transferred to the cue and induces behavioral 'wanting'. It is accompanied by high phasic dopaminergic firing in the core of the NAc (Flagel et al., 2011) and relies on the central-amygdala/nigral dopamine system (El-Amamy and Holland, 2006), which has also been implicated in attentional processing (Lee et al., 2006). Conversely, the goal-tracking behavior does not depend on striatal dopamine (Flagel et al., 2011). Selective breeding confirmed that animals who develop sign-tracking behavior in Pavlovian conditioning are characterized by high novelty-seeking and low self-inhibition, and exhibit particularly high dopamine release in the NAc in response to a Pavlovian cue (Flagel et al., 2011). Appetitive memories also seem to be more difficult to extinguish and are more resistant to updating in sign-trackers than goal trackers (Olshavsky et al., 2013).

The human analogue of these differences in reward learning is captured by Gray's Reinforcement Sensitivity Theory (Gray, 1991) which proposes that the extent to which appetitive stimuli activate the Behavioural Approach System (BAS) reflects a trait reward sensitivity (for reviews see Bijttebier et al., 2009; Corr, 2004). The BAS is conceptualized as a motivational system which primarily responds to stimuli indicating reward or non-punishment (e.g., relief, Corr, 2004). Subjects with high behavioral approach appear to be sensitive mainly to positive outcomes and to a lesser extent to the omissions of rewards, whereas subjects with low behavioral approach display a reduced response to rewards. High BAS sensitivity scores are related to individual impulsivity and are associated with substance (alcohol, tobacco, illicit drugs) abuse in clinical populations (Franken et al., 2006), as well as with high reactivity to alcohol cues and craving in non-clinical populations (Franken, 2002). Neural activity during reward processing can be modulated by individual differences in impulsivity (Martin and Potts, 2004) and in the total BAS score (including sub-scales 'Drive', 'Reward Responsiveness', and 'Fun Seeking'), with a positive correlation between the total BAS and the magnitude of $\mathrm{FMRI}$ response in the ventral striatum 
and the medial orbitofrontal cortex during the receipt of reward in a monetary incentive delay task (Hahn et al., 2009; Simon et al., 2010). This may be a sign of higher motivational salience assigned to monetary rewards in human subjects scoring high on BAS, which could be analogous to the aforementioned sign-tracking response in rats. Future investigations could test whether BAS scores are also related to the general and reward-modulated memory formation in humans.

Neural response to reward delivery is also modulated by other individual differences, including risk aversion (Tobler et al., 2007) and extraversion (Cohen et al., 2005), which may be related to dopaminergic transmission. In more detail, the presence of the D2 Taq1A allele associated with fewer D2 dopamine receptors populating the reward system (and thus with lower reward sensitivity) predicted inter-subject variability in reward-delivery-related activations in the medial orbitofrontal cortex, the amygdala and NAc: extraversion correlated with greater difference in activation for receipt versus non-receipt of reward, while the carriers of the A1 allele on the dopamine D2 receptor gene displayed a significantly reduced difference (Cohen et al., 2005). In fact, high striatal dopamine may enhance reward learning while possibly impairing learning from negative outcomes (Sharot et al., 2012), and vice-versa for dopamine depletion (Frank, 2011). Together, these results illustrate the role of genetic differences in dopamine receptor availability and personality traits in reward learning, however, more research is needed to verify how these may relate to the general function of dopamine in hippocampal memory formation.

All the above described differences in sensitivity to reward refer to paradigms where an extrinsic reward (usually money or food) is used. However, intrinsic motivation is another important individual factor that may bias responses to experimental reward manipulation in humans. Daniel and Pollmann (2010) found that perceived competence, which is presumed to be a predictor of intrinsic motivation, correlated with BOLD signal change in the NAc during the delivery of positive cognitive feedback (presentation of a green circle), while extrinsic motivation (as indicated by perceived pressure/tension reported in the Intrinsic Motivation Inventory; McAuley et al., 1989) 
predicted signal change in the NAc when monetary rewards were awarded. Similarly, Mizuno and colleagues (2008) showed a linear relation between academic achievement motivation scores and activity in bilateral putamen while the subjects awaited feedback in a working memory task posed as an intelligence test, suggesting that intrinsic motivation may affect reward network activity in a context-dependent manner. In tasks that deliver extrinsic rewards and thus rely on extrinsic motivation, such as a gambling task, BOLD signal in the prefrontal regions, amygdala, caudate and putamen was reduced in individuals with relatively low extrinsic but high intrinsic motivation during monetary reward delivery (Linke et al., 2010).

Intrinsic motivation may pertain to a large variety of individual aspirations, among which 'satisfaction of curiosity' and 'novelty' may represent canonical forms of intrinsic rewards. Novelty can thus be intrinsically rewarding, especially to individuals scoring high on novelty-seeking. A positive correlation between novelty-seeking trait and the magnitude of BOLD response was observed in the VTA when novel cues were presented (Krebs, Schott, \& Düzel, 2009), and subsequent memory for the novel cue was enhanced in those individuals regardless of the monetary reward offered, indicating that both the intrinsic value of novelty and the extrinsic value of monetary reward interact through dopamine activity to modulate memory. Curiosity on its own has been demonstrated to mimic extrinsic reward anticipation and novelty. For example, Kang et al. (2009) found that curiosity is associated with higher arousal (increased pupil dilation) and elevated striatal and prefrontal BOLD activity during anticipation of the outcome (in this case, the answer to an interesting quiz question).

As alluded to above and remarkably, the impact of monetary reward on memory was found to be more pronounced if the participant has little interest in the experimental task (Murayama and Kuhbandner, 2011; Murayama et al., 2010). Money can thus be used to boost both immediate and long-term memory for otherwise intrinsically unrewarding (uninteresting) information, but it has little effect on memory for information that is already intrinsically rewarding (Murayama and 
Kuhbandner, 2011). In an extreme situation, extrinsic incentives applied to an intrinsically motivating behavior may even override the positive effects of the latter on learning and performance (Deci et al., 1999; Murayama et al., 2010).

Paradoxically, the anticipation of high rewards may turn to be stressful and overly arousing, inducing threat of loss (Chib et al., 2012; Mobbs et al., 2009) and undermining potential learning gains. For example, in a foreign-language word learning experiment, Callan and Schweighofer (2008) presented Japanese participants with English words associated with either a high or low monetary reward for correct recall on a subsequent test performed several minutes later. Surprisingly, there was no significant effect of reward on recall performance. However, recall performance for highly rewarded words correlated negatively with individual state anxiety ratings, confirming that in sensitive subjects administering a test, even if highly rewarded, may also be associated with unfavorable emotional reactions, such as anxiety. High rewards may thus induce 'choking under pressure' and fear of loss (Mobbs et al., 2009). Resulting anticipatory anxiety reduces activation in striatal regions (Callan and Schweighofer, 2008; Chib et al., 2012) and could be mediated by serotonin, which potentially acts opposingly to dopamine in rewardmotivated behavior (den Ouden et al., 2013; Kakade and Dayan, 2002). Thus, under specific circumstances, extrinsic reward motivation can turn into punishment avoidance and impede learning. Such a switch in motivational drive may involve subcortical areas of the dopaminergic reward network where both approach and withdrawal behaviors can be initiated (Kravitz et al., 2012).

To sum up, the degree to which reward may enhance learning and memory depends on several individual factors, including personality traits such as impulsivity, extraversion and noveltyseeking traits, the level of intrinsic motivation to perform the task, as well as physiological state and personal preferences determining the valuation of the (extrinsic) reward. Experiments with motivated encoding show that individual reward responsiveness directly affects later recall, 
including circumstances when extrinsic reward may actually impair learning for some individuals. However, more research is needed to examine how individual differences assessed by personality questionnaires relate to the general function of dopamine in hippocampal memory formation. Understanding this relationship could be used for, e.g., personalization of educational and learning tools.

\section{Transfer of learned reward value}

In some experimental manipulations, a generalization of conditioned reward response to stimuli that are novel or have not been directly associated with reward is observed. This is referred to as transfer of incentive salience. Often, the generalized conditioned response is not accompanied by declarative memory enhancement but entails increased response vigor or choice bias, without awareness of the underlying indirect reward-association (Wimmer and Shohamy, 2012, 2011). The phenomenon can be viewed as spreading of reward motivation to cues that do not possess any incentive value and its implications are of interest for the fields as varied as decision making and education.

Below, we describe those circumstances under which reward motivation transfers across various dimensions (perceptual similarity, common association, time, etc.) and produces biases in perception and behavior (section 3.1) or enhances declarative memory (section 3.2), followed by a general discussion.

\subsection{Reward biases perception and behavior}

One aspect of learning which has been observed to varying degrees in fear and in reward learning concerns generalization of the acquired emotional and/or motivational values from the experienced stimuli to related, but new stimuli (see section 1.3 above, Box 1 and Figure 3). Here, value denotes relevance in terms of a behaviorally significant outcome, which can be punishing or rewarding. Transfer of reward value is assessed in this context as transfer of motivation where motivation has both activational and directional functions (Salamone and Correa, 2012). 
Activation is related to stimulus-unspecific invigoration of response (i.e., working harder to obtain reward), typically gauged by response rate (Niv et al., 2007), and direction is related to specific response biases, typically assessed by choices. Generalization of acquired values can diffuse along gradients of similarity (similarity-based stimulus generalization), as well as via more complex relations, such as inferences, associations, or instrumental conditioning (e.g., Pavlovian-toIntrumental Transfer), as we discuss in more detail below.

\subsubsection{Similarity-based stimulus generalization}

Generalization of reward conditioning involves the spreading of reward value from one conditioned stimulus (CS+) to another stimulus that has not been paired with reward. Much like for aversive conditioning (e.g. Dunsmoor, Kragel, Martin, \& Labar, 2013; Dunsmoor, Martin, \& LaBar, 2012; Dunsmoor, Mitroff, \& LaBar, 2009; Maren, Phan, \& Liberzon, 2013), generalization of reward conditioning may partly rely on physical properties of the stimuli. Conditioned responses (CRs) can extend to stimuli that resemble the CS+ along some basic perceptual dimensions, such as tone pitch, size, or color (Honig and Urcuioli, 1981), or generalize to stimuli sharing more abstract characteristics, such as belonging to a specific visual category like 'cars' or 'flowers' (Bhatt et al., 1988).

Ever since Pavlov discovered that dogs conditioned to salivate to a ringing bell would also respond to a ring of a similar pitch (Bouton, 2007; Pavlov, 1927), generalization of the CR has been studied in various species using transfer tests - i.e. measuring the response rate to new stimuli resembling previously conditioned stimuli. Results from such experiments can be illustrated by a stimulus generalization gradient which describes the rate at which the CR declines as the stimulus is changed, along some discriminative dimension, from the CS+ to the non-predictive CS- (Bouton, 2007). Importantly, the shape of the generalization gradient can be affected by the learning context. A steeper gradient reflects highly specific discrimination can typically result from discrimination training with two stimuli (a CS+ and a CS-; Jenkins \& Harrison, 1960), or from the 
type of generalization testing procedure adopted (e.g., although generalization gradients could be flat for both CS+ and CS-, extinction may be faster for stimuli more similar to the CS+; Vervliet, Iberico, Vervoort, \& Baeyens, 2011).

Generalization of reward conditioning has some distinct properties that are well replicated across experiments and species: (1) physical similarity between stimuli facilitates generalization; (2) generalization is enhanced for dimensions of the stimulus with which the organism has little experience (i.e., novel stimuli); (3) in animals, generalization is largely independent of the type of stimuli or behavioral responses, so that discrimination about food items or arbitrary tokens or even potential mating partners will yield the same results; (4) associating several perceptually different exemplars of stimuli rather than one single exemplar with the same rewarding outcome will lead to broad generalization gradients, while using similar CS+ and CS- produces gradients along the discriminative stimulus dimension that are narrower, reflecting finer discrimination; (5) the 'peak shift', also known as response bias, occurs when training includes both a CS+ and a CSand refers to the fact that the gradient peak around which CR is maximal is not observed for the discriminative stimulus dimension equal to that of the CS+ but is rather located farther away from the CS-; (6) the 'peak-shift' is reduced with longer testing runs, due to extinction of the conditioned response (Ghirlanda and Enquist, 2003).

A recent experiment by Kahnt et al. (2012) has demonstrated the 'peak-shift' in human volunteers. Subjects were first trained on Gabor patches of two different orientations, one CS+ and one CS- (e.g. $39^{\circ}$ and $\left.51^{\circ}\right)$ in a fully reinforced conditioning procedure (rewarded with 30 and 0 points, respectively). Next, they judged whether gratings with similar orientations to those conditioned would predict reward or not. The behavioral responses (i.e., orientations classified as predicting reward) revealed a shift in the peak of the generalization gradient away from the CS(for illustration see Figure 3A). Importantly, fMRI data acquired simultaneously revealed that the magnitude of ventral striatal activation in response to stimuli that had never been paired with 
reward correlated with prediction-error (i.e., the difference between the expected and actually received reward), suggesting generalization of value representations from the previously rewarded CS+. Moreover, functional connectivity between the ventral striatum and the hippocampus predicted individual differences in the width of generalization. Because projections from the hippocampus to the ventral striatum can gate dopaminergic activity in the midbrain (Figure 1; Lisman \& Grace, 2005), the authors suggested that dopamine may control the width of generalization (depicted in pink in Figure 2B). Partial support for this thesis comes from studies on the generalization of fear-conditioned response in humans. In healthy controls the gradient of generalization correlated with the magnitude of BOLD response in the VTA while the response in patients with generalized anxiety disorder was indiscriminately high in a generalization test (Cha et al., 2014), suggesting overgeneralization of fear CR.

[Figure 3 about here]

As mentioned above, generalization of conditioned aversive and approach responses share some aspects in terms of the associative dimensions along which new information may inherit a conditioned value. However, rewarding and aversive outcomes have different effects on generalization. Wider generalization gradients for aversive information may be critical for survival by allowing the rapid detection of potential threats in the environment (at the expense of more false alarms), while missing potential rewards may not be so costly. Schechtman and colleagues (2010) directly tested the hypothesis that when both punishment and reward are present, the punishers produce stronger generalization, as measured by wider generalization gradients. Human participants first learned to associate loss or gain of monetary outcomes with two distinct auditory stimuli. In the generalization test, the subjects performed a perceptual classification task on different tones, including the conditioned ones. Classification of the stimulus previously paired with a negative outcome (loss) resulted in wider generalization gradients even when compared to the rewarded stimulus with a reward value twice as large as the loss. Thus, as compared to 
positively-valenced associations, negatively-valenced associations generalized more to less similar situations. Note that this behavioral strategy corresponds to an 'irrational' choice in this task, because the wider the generalization, the more money participants lost. This result parallels the findings of Kahnt et al. (2012), where in a modified reinforcement learning model that included a generalization parameter, the gradient of the negative prediction error adjustment (in case of no reward when one was expected) was wider than that of a positive prediction error.

\subsubsection{Generalization based on association or acquired equivalence}

Unlike similarity-based stimulus generalization tasks, in which generalization spreads along relevant sensory dimensions of the CS+, incentive salience transfer may diffuse across physically different stimuli that are linked through direct association or via a common stimulus or outcome.

Transfer of learned value to stimuli that have a relational representation in memory has been studied using sensory preconditioning (Hall, 1996). Sensory preconditioning involves three phases: (1) pairing of stimuli (e.g. by presenting the two stimuli in close temporal proximity, or by pairing them with the same outcome), (2) conditioning of one member of the pair, and (3) testing the response to both the conditioned stimulus and the stimulus initially paired with the conditioned stimulus (Figure 3B). Sensory preconditioning can reliably endow the paired stimulus with the ability to elicit a similar response as the conditioned stimulus, even though it has never been directly associated with a reward outcome. Phases 1 and 2 in the sensory preconditioning procedure can also be swapped and yield a similar transfer of conditioned response, a procedure called backward or second-order conditioning. Wimmer and Shohamy (2012) have studied the neural bases of stimulus preconditioning in humans. In the first phase of the experiment, 6 neutral stimuli (body parts, faces, scenes, 2 of each category) designated as S1, and 6 abstract patterns designated as $\mathbf{S} 2$, were presented in temporal succession, so as to create incidental associations between elements of 6 S1-S2 pairs. Next, in a Pavlovian conditioning procedure, half of the abstract patterns were followed by monetary reward ( 3 different CS+ previously paired 
with a scene, a face and a body part) or nothing ( 3 remaining cues as CS-). During a behavioral decision phase, participants were asked to choose between two stimuli (two S1 or two S2) for a possible monetary win. While participants tended to choose the S2+ items over the S2- items, supporting successful reward learning, a bias to choose the S1+ over the S1- items varied markedly across participants and was not accompanied by conscious memory of the association. A decision bias was thus operationalized as the tendency to choose S1+ over S1- stimuli (see Figure $3 \mathrm{~B})$. The $\mathrm{fMRI}$ data revealed that the magnitude of this bias correlated with the level of reactivation in selective visual regions coding for the distinct S1 during the reward phase, when the S2 stimuli (but no S1 stimulus) were presented, thus demonstrating that the transfer of reward value relied on the preestablished S1-S2 associations. Moreover, the activation in the caudate correlated with that of the hippocampus as a function of the behavioral decision bias (orange in Figure $2 \mathrm{~B}$ ), suggesting that the functional connectivity between the caudate and the hippocampus may, at least partly, mediate value-based decision bias.

Generalization may also be achieved by pairing two stimuli with the same outcome or by presenting each with a common accompanying feature, a procedure known as 'acquired equivalence'. In acquired equivalence paradigms, dissimilar stimuli (S1 and S2) will be treated similarly if they predict the same outcome, so that any pairing of S1 with a new outcome will transfer to S2. In an acquired equivalence task, participants are thus trained implicitly via trial and error or via explicit associative training to memorize a set of overlapping relationships (Zeithamova et al., 2012). Another experiment by Wimmer et al. (2012) demonstrated that generalization through acquired equivalence can also influence decisions in humans. The researchers used a probabilistic reinforcement learning variant of the acquired equivalence task where participants learned the reward value of one of four faces by choosing one at a time. The faces formed two pairs within which the faces predicted monetary reward that participants accumulated across the trials with equal probability. On a given trial the outcome was displayed only for one chosen face. Participants were not explicitly aware of the underlying reward 
probability structure. The behavioral choices in terms of reward prediction error from trial to trial were modeled on two reinforcement learning models - one that updates the value of the partner face image as the value of the other (probability-equivalent) face is learned, and one which is blind to the task's correlational structure. The former model turned out to fit the behavior with superior accuracy. A specific regressor that accounted for the difference between the two models - i.e., the additional explanatory power of the generalization of learned value from one face to its partner face - correlated with the BOLD response in a cluster centered on the ventral striatum. In addition, this area was functionally connected with the hippocampus, suggesting that the hippocampus supports generalization between option values that are learned over time by trial and error (in red, Figure 2B).

\subsubsection{Pavlovian-to-Instrumental Transfer (PIT).}

Reward-value memory and associated energizing effects of reward motivation may linger and transfer to other tasks producing, for instance, speeded responding to previously rewarded items in a non-rewarded task that immediately follows a reward learning procedure (Madan et al., 2012). According to Berridge's notion of incentive salience, the reward-conditioned cue can prime further consumption by associative spread of incentive salience among linked representations, so that it is difficult to stop at just one small treat (Berridge, 2012). Importantly, incentive salience is generated anew each time the CS+ is perceived, and depends on the current state of the organism (hunger, thirst, etc.), as well as on mesolimbic dopamine levels (Berridge, 2007). Such a spread of incentive salience may be induced by a procedure called Pavlovian-to-Instrumental Transfer (PIT). It describes the capacity of an appetitive pavlovian stimulus (CS+) to elicit or increase the vigor of an ongoing behavior in an instrumental conditioning procedure. Two main types of PIT have been distinguished: general and specific (Balleine and Killcross, 2006; Holmes et al., 2010). In general PIT, the CS+ enhances any appetitive instrumental response, even when the instrumental response is associated with a different outcome, whereas in specific PIT, the CS+ 
associated with a certain outcome only enhances an instrumental response associated with the same outcome (Figure 3C). Specific PIT can be interpreted as activation of the learned behavioral sequence associated with the outcome predicted by the presented CS+ (the behavioral chaining account, Holmes et al., 2010; van den Bos et al., 2004). General PIT can be thought of as an expression of the transfer of incentive salience and the associated energy expenditure to obtain reward, from the CS+ to the ongoing behavior (Berridge, 2007; Holmes et al., 2010), which is accompanied by a change in the motivational state (Holland and Gallagher, 2003).

A first neuroimaging study to provide behavioral and neural evidence for human PIT was reported by Talmi et al. (2008). Participants first learned the relationship between a certain visual background presented together with a specific sound (CS+) that revealed a coin (monetary reward) after a button press, then another visual-auditory stimulus which always failed to deliver a reward (CS-), and a baseline stimulus associated with no reward opportunity (Figure 3C). Next, in the instrumental phase, the subjects practiced squeezing a hand grip with maximum force which, if done within two specific time windows (unknown to the participant) within a 12s-long block, delivered the monetary reward. This phase was presented on a different, uniform visual background and the image of a coin symbolizing reward was accompanied by the sound of a waterfall. The transfer test followed, in which the Pavlovian CS+, CS- and the baseline cue replaced the instrumental visual background, and the auditory stimuli from the conditioning phase were superimposed on the waterfall sound used in the instrumental phase. Instrumental response (number of hand grip squeezes over $50 \%$ of participants' maximal force) was significantly higher in the presence of the CS+ than of the other stimuli, and this effect subsided with time, demonstrating extinction. The fMRI results showed that PIT in humans implicated brain areas analogous to those reported in rodents, namely the NAc and the amygdala (in yellow, Figure $2 \mathrm{~B})$, and that BOLD signal in these areas correlated positively with the instrumental response. In addition, PIT was stronger in participants who seemed to be aware of their increased vigor of response when the CS+ was present on the screen, which indicates that awareness of the 
manipulation may be an influential aspect of conditioning studies in humans. It is worth noting that, in this experiment, pavlovian and instrumental outcomes were the same so that both specific and general mechanisms may have contributed to the observed PIT effect.

\subsection{When reward motivation enhances declarative memory}

\subsubsection{Incidental learning during state of reward motivation}

As detailed in the previous section, reward transfer can boost non-declarative memory formation. There is some evidence that such generalization processes may also influence declarative memory. A study by Wittmann et al. (2011) showed that when the semantic category of a word predicted reward, the memory for these reward-predicting words was increased. But when a perceptual aspect of the word (font color) was the reward predictor, the memory for word identity was no better in the rewarded than in the non-rewarded condition. This suggests that although announcement of reward in itself may increase learning of reward-predicting stimuli, declarative memory enhancement may more selectively affect the reward-predicting dimensions of the stimulus, perhaps depending on the required depth of processing. On the other hand, some studies suggest that during reward anticipation, encoding may be enhanced not only for rewardrelevant cues and all information related to the prediction of reward, but also for unexpected events irrelevant to it. For example, Murty and Adcock (Murty and Adcock, 2014) showed that in highly-rewarded trial blocks but not in low-rewarded ones, the presentation of a non-target oddball image while anticipating the appearance of the target image resulted in above-chance memory for these oddballs in an immediate recognition memory test (Figure 4A). Furthermore, the $\mathrm{fMRI}$ data revealed that during high-reward blocks, the left hippocampus was more active in response to the oddballs (referred to as expectancy violations) than during low-reward blocks. Moreover, on a trial-by-trial basis, the magnitude of VTA activation during reward cue presentation positively correlated with later hippocampal response to the oddball presentation. The authors interpreted their finding as an effect of reward on salience processing by the 
hippocampus, enacted directly via hippocampal-VTA interactions (Lisman and Grace, 2005; Lisman et al., 2011) (Figure 1) or, as indicated by the results of their connectivity analysis, via cortical regions, including the prefrontal cortex and visual cortices, to which the VTA also projects (Murty \& Adcock, 2014 in dark blue, Figure 2A).

[Figure 4 about here]

According to the penumbra hypothesis of dopamine action on the MTL structures (Lisman et al., 2011; see section 2.3), the release of dopamine enhances hippocampus-dependent memory formation (and later transformation) for all events encoded (or retrieved) during the time window of its availability at the hippocampal synapses ( up to $30 \mathrm{~min}$ in the rat, Bethus et al., 2010). Therefore, presentation of a reward (or a novel stimulus) may affect events occurring several seconds to minutes afterward, during the time when the evoked dopaminergic input from the VTA reaches the hippocampus.

Gruber and colleagues (2014, using a paradigm similar to Kang et al., 2009) found that a state of high curiosity (i.e., a state of intrinsic reward motivation) was associated with NAc and VTA activation and led to better free recall memory for trivia questions the participants were curious about, $1 \mathrm{~h}$ after the encoding. Furthermore, this experiment directly tested the penumbra hypothesis by showing participants faces during the answer anticipation period. Incidental encoding of the faces tested immediately after the experiment revealed strong individual differences, whereby activity in the VTA and high VTA-HPC connectivity correlated with the memory advantage. Moreover, face recognition displayed some advantage for faces encoded during a state of high curiosity when tested one day later. These results suggest that dopaminergic activity modulates peri-encoding processes and enhances memory formation for both reward-related and incidentally perceived information.

Also consistent with the penumbra hypothesis, Fenker et al. (Exp. 2, 2008) demonstrated that exploring novel natural scene photographs for $10-\mathrm{min}$ as opposed to exploration of familiar 
scenes prior to encoding of a list of words, enhanced subsequent free recall of the words. The effect was observed in a test several minutes after encoding, even though the participants were presented with familiar scenes immediately after the novelty exploration in order to equalize the level of arousal at testing between the two groups. Furthermore, free recall performance correlated with parahippocampal and prefrontal activation in the novelty-context group only, while SN/VTA activation during scene viewing (novel/familiar vs. fixation) correlated with later free recall of studied words in both groups. In a second related experiment, Fenker and colleagues (Exp. 1, 2008) found that the novelty intervention (viewing novel pictures) also improved recognition memory, of subsequently studied words, both at immediate and delayed (24h) testing. This provides first evidence that the effect of novelty-induced dopamine release outlasts the evoking event and spreads to nonselectively facilitate encoding even 15-30 minutes later (see also Schomaker and Meeter, 2015 for a review of effects of novelty on memory and cognition ). Taken together, these studies suggest that stimuli or features presented concurrently with the reward-predicting cues are remembered better especially if goal-relevant, while the general state of reward motivation may enhance encoding of those stimuli or features, whether goal-relevant or not.

Additional support for the role of increased reward motivation on memory comes from an experiment by Mather and Schoeke (2011), in which a potential reward was signaled and followed by a cartoon image that required a speeded motor response in order to win the trial. On an immediate free recall test, those images were better remembered for which the reward was actually won (delivered), suggesting that reward delivery rather than the mere anticipation of a reward may enhance memory consolidation for predictive cues, particularly when performance uncertainty is involved. In addition, memory enhancement was observed for images appearing up to two trials after the winning trial. This observation adds to other evidence demonstrating that positive feedback during learning may act as an internal reinforcer, instilling a sense of intrinsic reward motivation (subjective achievement) which improves learning on the current and 
subsequent trials. This is true for declarative (Mather and Schoeke, 2011) and procedural (Wächter et al., 2009) memory, as well as in perceptual learning (Seitz and Watanabe, 2005), where even performance-unrelated (i.e., fake) feedback produces such results (Shibata et al., 2009).

\subsubsection{Reward-related effects on (peri-encoding) memory integration}

Memory integration into schemas pinpoints the particular interaction between not only the hippocampus and the VTA, as reported in previous sections, but also the prefrontal cortex. In particular, the ventro-medial part of the prefrontal cortex (vmPFC, mPFC in rodents) in humans has been associated with at least two specific functions which may contribute to the effects of reward motivation on memory integration. Firstly, as a recipient of dopaminergic projections from motivational value-coding neurons in the ventromedial $\mathrm{SNpc}$, it is driven by value or salience (Bromberg-Martin et al., 2010b) and is involved in guiding decisions based on value comparison (De Martino et al., 2013). BOLD signal in the vmPFC in tasks involving choice selection reflects the benefit of the action taken as well as the opportunity cost of the unchosen option (Rushworth et al., 2011). Secondly, human vmPFC is implicated in the use of schemas during encoding and periencoding consolidation. For example, van Kesteren and colleagues (2010) observed that enhanced vmPFC-hippocampus connectivity facilitated the encoding of new information when there was no prior schema, while prior knowledge about an event reduced hippocampal-vmPFC connectivity. A related putative role of VmPFC in human hippocampal memory processes is that of resolving conflict during accommodation of new memories into existing memory schemas, as well as in retrieval of memories already incorporated into a schema (Preston and Eichenbaum, 2013). For instance, Kumaran and colleagues (2009) observed that co-activation in the vmPFC and the hippocampus correlated with the probability of performance success in a task requiring the extraction of patterns underlying the abstract structure of a weather-prediction learning task. Functional correlation between the hippocampus and VmPFC was also observed in the 
generalization of the learned complex abstract rule to a set of novel stimuli. These and other data focusing on memory schemas offer new insight into the potential role of dopamine in memory integration. However, future studies are needed that would specifically target the implication of the dopaminergic reward system by using dedicated behavioral and fMRI designs, or pharmacological and molecular imaging approaches (Takahashi et al., 2012).

Generalization of memory may involve the recombination of encoded associations. This type of generalization is observed in associative inference tasks, in which participants are typically exposed to pairs of stimuli with different reward contingencies, and are expected to infer a relationship among them (Figure 4B). For example, participants are rewarded for choosing $A$ in a pair of stimuli containing $A$ and $B$ presented together $(A+, B-)$, and for choosing $B$ in a pair containing stimuli $B$ and $C(B+, C-)$. Stimuli $A$ and $C$ are then presented together, thus representing a new, never seen stimulus pair, and the participants are expected to infer that $A$ is the correct choice. Shohamy and Wagner (2008) demonstrated that the process of inference is supported by a cooperative interaction between the hippocampus and the VTA. Specifically, the fMRI results showed that better generalization was preceded by a greater increase in hippocampal and midbrain activation between early and late learning of the reward contingencies. This study thus suggests that the hippocampal-VTA loop may facilitate cross-item integration during encoding, in addition to enhancing episodic memory for novel events (Lisman \& Grace 2005). Namely, hippocampal-VTA interactions would facilitate rapid retrieval during encoding leading to cross-item integration, or what has been referred to as peri-encoding consolidation (Cohen et al., 2014). This role of the dopaminergic projections to the hippocampus likely has little to do with hedonic aspects of reward. Indeed, in Shohamy \& Wagner's study, the subjects were equally compensated for their participation irrespective of performance and did not receive feedback about the correctness of their choices in the test phase. 
The associative inference paradigm and this particular study illustrate well the implied role of dopamine as the link between anticipatory reward motivation and memory formation processes. While dopamine levels modulate trial-and-error learning from positive feedback (in this case, during the initial training phase), they may also explain the observed performance differences in a related transitive inference task (Frank et al., 2006). Frank and colleagues (2006) have even interpreted this task as essentially an implicit-memory task relying on the basal ganglia rather than the MTL memory system. Some evidence, however, suggests that the activation of the hippocampus in this task scales with the degree of relational processing necessary for inference judgments, with less activation for more relationally distant items (Zalesak and Heckers, 2009). Yet another study showed that hippocampus and caudate interact cooperatively to facilitate successful inference (Moses et al., 2010). Since the involvement of dopaminergic pathways is strongly implicated in this task, an unanswered question that remains is whether increasing dopamine levels in healthy participants by means of reward, novelty or curiosity, or pharmacological manipulation, could facilitate inference.

\subsubsection{Reward motivation transfer in recollection and memory transformation}

Recent theories on reconsolidation propose that long-term memory formation implicates memory recollection, because the hippocampus links encoded information to reactivated remembered information in a continuous cycle of retrieval and learning (Alberini, 2011; Forcato et al., 2007; McKenzie and Eichenbaum, 2011; Stickgold and Walker, 2013). In this context, the circuits modulated by new learning are the ones that are activated by the reminder of a remembered (e.g. semantically) similar event (McKenzie and Eichenbaum, 2011). If reward enhances memory consolidation (see sections 2.3 and Box 2), one ensuing question is whether reward-associated memories interplay with new learning differently from neutral memories. For example, does recollection of a reward memory bring about the release of dopamine and facilitate new memory 
consolidation (Lansink et al. 2009)? Or is the reward memory so resistant to forgetting that it actually impairs memory updating?

In a behavioral experiment, Madan and colleagues (2012) have demonstrated that after a reward conditioning procedure, reward-associated items are more readily recalled in a non-rewarded task involving word judgment than non-rewarded words. Critically, they also found that the memory of the reward value attached to the conditioned words later interfered with new learning, when participants were given lists of 9 words followed by a distractor task and immediate free recall. In the recall phase, highly rewarded words produced the highest number of intrusions defined as a failure of contextual discrimination, i.e., recall of items that have previously been studied but did not come from the immediately preceding word list. The authors suggested that this may be evidence for reward value impairing contextual binding and proposed a value-based interference hypothesis according to which learned value may impair some facets of memory (such as in this case, contextual binding). This result would suggest that a rewardvalue memory generalization may have a negative effect on source memory information in later learning.

However, Kuhl and colleagues (2010) found that although older rewarded memories are remembered better than newer ones, reward level did not modulate resistance to retroactive interference. In this neuroimaging experiment, the participants studied pairs of cues with associates $(A B)$, the memory of which was subsequently tested in a retrieval run, and followed by another encoding run presenting a part of the initially used stimuli as repeated cues with novel associates (AC). Such a manipulation produces retroactive interference leading to the deterioration of memory for the original $A B$ pair (Figure $4 C$ ). In the variant used by Kuhl et al. (2010), initial $A B$ pairs were rewarded with either high or low monetary compensation announced by a cue presented before each pair. This reward manipulation on the first pair to be learned resulted in memory enhancement for the highly rewarded $A B$ pairs (in a post-test outside the 
scanner), but reward did not affect the degree of interference due to later AC learning (in immediate post-encoding tests). Importantly, activity in reward-responsive regions - the ventral striatum and vmPFC - during $A C$ encoding was predictive of later memory for the AB pair. This positive correlation was significant for high-reward pairs only. Moreover, the magnitude of the subsequent memory effect in the hippocampus and the ventral striatum were positively correlated. The authors concluded that hippocampal pattern completion processes reactivated the previously encoded $A B$ pairs and their associated reward context during $A C$ encoding, and that this reactivation protected the $A B$ memories against forgetting due to interference. In addition, higher hippocampal BOLD response during AC learning was associated with lower retroactive interference in the subsequent test. Although the behavioral results in this study showed that highly rewarded pairs did not interfere with new learning more than low rewarded ones, one may want to ask what would happen if a longer delay is used (including a night of sleep, see Box 2) between early reward learning and new associative learning. Is it possible that previous reward association would lead to reduced or no interference, while at the same time making the learning of new partially overlapping relations easier? A better understanding of effects of prior reward associations on future learning awaits future investigation.

\subsection{Discussion}

Traditionally, memory processes in the medial temporal lobe (Eichenbaum, 2004; Squire \& Wixted, 2011) and processes in the striatum (Frank and Claus, 2006) have been hypothesized to work rather independently from each other, leading to the division of their functions into, respectively, declarative and non-declarative memory. The data reviewed above support instead that both systems have strong interactions and may mediate memory generalization processes (Delgado and Dickerson, 2012; Packard and Goodman, 2012; Poldrack and Foerde, 2008; Sadeh et al., 2011). The data support the view that the hippocampal interactions with the VTA and VmPFC are mandated for declarative memory enhancements, while the hippocampal-striatal (NAc and caudate) interaction is observed when reward value transfer results in non-declarative effects. 
On the other hand, it is not ruled out that more complex, competitive mechanisms are at work between the MTL and the striatum-based memory systems. It has been found that in a probabilistic reward learning task, later memory for cue identity (cartoon picture) negatively affected the learning of the reward predictive property based on cue-frame color (Wimmer et al., 2014). Hippocampal-striatal connectivity was higher on those subsequently remembered trials. Therefore, since on a trial-by-trial basis, better memory is associated with worse reward-based updating, it is possible that in such a setting episodic encoding may compete with reinforcement learning or that the two tasks compete for limited working memory resources. At the same time, recognition memory was higher for objects presented in the chosen vs. non-chosen frame, suggesting the influence of attentional and decision mechanisms.

The distinction between circumstances in which reward value generalization promotes selective enhancement in explicit memory and in which it results in implicit biases and effects on motor or habitual responses, is not quite clear. Studies on the interactions between the two memory systems in humans suggest that increased functional connectivity between the prefrontal cortex and the striatum are a hallmark of the emergence of explicit knowledge (Rose et al., 2010), while others stipulate that implicit memory may simply be viewed as sub-threshold activation of the same neural circuit (the MTL) as in explicit declarative memory (Johnson et al., 2009). For the sake of optimal use of reward motivation in educational settings, it is necessary to extend current paradigms that reliably produce conditioned reward response, with a subsequent task of declarative memory formation that may include some previously rewarded elements.

\section{Conclusion}

This review brings together data from recent research on the memory-enhancing effects of reward and on different forms of transfer of learned reward values in humans. First, we show that the influence of reward on memory critically depends on dopamine and outlasts the mere event that elicits dopaminergic activity, leading to enhancement of memory formation and energization 
of instrumental behavior. Rewarding cues or feedback elicit this effect even without awareness or subject's intention. As a result, positive feedback stimulates learning seconds after the learning episode has been presented, while novelty exploration may induce a memory-stimulating neuromodulatory state that can last at least several minutes. Reward-value transfer in memory (discussed in section 3) raises the exciting question of whether reward value generalizes from items specifically paired with reward to those only potentially predicting reward (based on perceptual or semantic similarity, or some other relations).

Secondly, the above reviewed neuroimaging studies suggest that temporally-correlated activity in the hippocampus and in regions of the dopaminergic circuit (striatum, VTA) may foster the generalization of reward value from learned associations in the human brain as well as formation of mnemonic links. The functional connectivity between the reward circuit and the hippocampus may come to play a more general role in learning and especially in transferring learned value to new decisions. Future research ought to distinguish whether the observed activation is related to reward-anticipation attributed to the dopaminergic activity of the VTA or rather a more general learning function that drives generalization of value (Box 2). And if so, could other events triggering dopamine release, such as for example listening to favorite passages in a musical record (Salimpoor et al., 2011), produce a state that favors memory formation?

We note that anticipatory-reward motivation does not equal dopamine release because "motivational processes interact with mechanisms related to emotion, learning, and other functions, and that there is not a precise point-to-point mapping between behavioral processes and neural systems" (Salamone and Correa, 2012). Yet, our intention was to bring together the literature on reinforcement learning and associative memory formation because some commonalities appear to be involved. This brings us to the hypothesis that dopamine may code for an "episodic" prediction error because the hippocampus and midbrain dopamine neurons not only respond to violations in reward expectation but also to deviations in the content of an 
experience, such as signaling the occurrence of a novel and unexpected event, or the nonoccurrence of an expected one (Lisman and Grace, 2005; Wimmer and Shohamy, 2011).

We furthermore noted that the motivating effect of reward also partly depends on personality traits, preferences as well as physiological state (section 2.4). Paralleling rodent studies, human research still needs to clarify whether and how individual differences in dopamine receptor expression, trait novelty-seeking or other correlates of dopamine sensitivity contribute to performance not only on extrinsically-rewarded tasks but also on associative inference and other generalization tasks. It is also important to test whether performance on such tasks can be influenced by extrinsic triggers of dopaminergic activity (such as monetary reward, positive social feedback, surprise, funny images or pharmacological manipulation). The case of extrinsic reward is of special interest as it may also produce loss-avoidance behavior and anxiety. As a result, and paradoxically, extrinsic reward may hinder learning in some cases, while facilitating it in others.

It seems particularly relevant for research in education to determine how to optimize extrinsic reward such that it maximizes memory benefits while respecting individual sensitivity to rewards and punishment. As some experiments in US-based primary and secondary schools show, simply paying students for good grades is not a wise recommendation (Flannery, 2011), indicating that to foster learning the reward structure needs to be more varied and complex. Much may be learned from how the video game industry uses extrinsic rewards to captivate the players, and insure at least the kind of time on task that is a pre-requisite of any learning (Howard-Jones et al., 2011). In the future, projects investigating whether and how novelty exploration, emotional arousal or extrinsic reward may boost lon-term memory formation, especially for material that does not inspire curiosity or intrinsic motivation, could help translate the present body of neuroscientific knowledge to practical applications. A first animal demonstration showed that novelty exploration indeed can facilitate consolidation in subsequent declarative memory formation and lead to improved recall 24h later (Salvetti et al., 2014). Similar effects of novelty exploration in humans, 
have so far been reported for immediate memory tests (Fenker et al., 2008; Schomaker et al., 2014). Also in this domain, there is preliminary evidence that transfer in training of some skills may depend on individual differences in prefrontal dopamine availability. Playing a first-person shooter video game every day for 3 weeks improved cognitive flexibility in individuals with the Val/Val variant of the COMT gene (i.e. lower prefrontal dopamine, cf. section 2.4) to a greater extent than in those with the Met/-polymorphism (Colzato et al., 2013). This emphasizes the need in future studies to take into account the role of individual differences in explaining the relationship between training and transfer, as the same intervention may not be of equal benefit to all.

\section{Acknowledgements}

This work was supported by the National Center of Competence in Research (NCCR) Affective Sciences financed by the Swiss National Science Foundation ( $n \circ 51 N F 40---104897$ ) and hosted by the University of Geneva, plus individual project grants from the Swiss National Science Foundation (No 100014_140676 to DB; No 320030_135653 to SS). 


\section{References}

Abe, M., Schambra, H., Wassermann, E.M., Luckenbaugh, D., Schweighofer, N., Cohen, L.G., 2011. Reward improves long-term retention of a motor memory through induction of offline memory gains. Curr. Biol. 21, 557-62. doi:10.1016/j.cub.2011.02.030

Adcock, R.A., Thangavel, A., Whitfield-Gabrieli, S., Knutson, B., Gabrieli, J.D.E., 2006. Rewardmotivated learning: mesolimbic activation precedes memory formation. Neuron 50, 507-17. doi:10.1016/j.neuron.2006.03.036

Alberini, C.M., 2011. The role of reconsolidation and the dynamic process of long-term memory formation and storage. Front. Behav. Neurosci. 5, 1-10. doi:10.3389/fnbeh.2011.00012

Alcaro, A., Panksepp, J., 2011. The SEEKING mind: primal neuro-affective substrates for appetitive incentive states and their pathological dynamics in addictions and depression. Neurosci. Biobehav. Rev. 35, 1805-20. doi:10.1016/j.neubiorev.2011.03.002

Anderson, B. a, Laurent, P. a, Yantis, S., 2011. Value-driven attentional capture. Proc. Natl. Acad. Sci. U. S. A. 108, 10367-71. doi:10.1073/pnas.1104047108

Ballarini, F., Moncada, D., Martinez, M.C., Alen, N., Viola, H., 2009. Behavioral tagging is a general mechanism of long-term memory formation. Proc. Natl. Acad. Sci. 106, 14599-14604. doi:10.1073/pnas.0907078106

Balleine, B.W., Killcross, S., 2006. Parallel incentive processing: an integrated view of amygdala function. Trends Neurosci. 29, 272-9. doi:10.1016/j.tins.2006.03.002

Baudonnat, M., Huber, A., David, V., Walton, M.E., 2013. Heads for learning, tails for memory: Reward, reinforcement and a role of dopamine in determining behavioral relevance across multiple timescales. Front. Neurosci. 7, 1-14. doi:10.3389/fnins.2013.00175

Ben-Yakov, A., Dudai, Y., 2011. Constructing realistic engrams: poststimulus activity of hippocampus and dorsal striatum predicts subsequent episodic memory. J. Neurosci. 31, 9032-9042. doi:10.1523/JNEUROSCI.0702-11.2011

Bermudez, M. a, Schultz, W., 2010. Responses of amygdala neurons to positive reward-predicting stimuli depend on background reward (contingency) rather than stimulus-reward pairing (contiguity). J. Neurophysiol. 103, 1158-70. doi:10.1152/jn.00933.2009

Berridge, K.C., 2007. The debate over dopamine's role in reward: the case for incentive salience. Psychopharmacology (Berl). 191, 391-431. doi:10.1007/s00213-006-0578-x

Berridge, K.C., 2012. From prediction error to incentive salience: mesolimbic computation of reward motivation. Eur. J. Neurosci. 35, 1124-1143. doi:10.1111/j.1460-9568.2012.07990.x

Berridge, K.C., Robinson, T.E., 2003. Parsing reward. Trends Neurosci. 26, 507-513. doi:10.1016/S0166-2236(03)00233-9 
Bethus, I., Tse, D., Morris, R.G.M., 2010. Dopamine and Memory: Modulation of the Persistence of Memory for Novel Hippocampal NMDA Receptor-Dependent Paired Associates. J. Neurosci. 30, 1610-1618. doi:10.1523/JNEUROSCI.2721-09.2010

Bhatt, R.S., Wasserman, E.A., Reynolds Jr, W.F., Knauss, K.S., 1988. Conceptual behavior in pigeons: Categorization of both familiar and novel examples from four classes of natural and artificial stimuli. J. Exp. Psychol. Anim. Behav. Process. 14, 219-234. doi:10.1037/00977403.14.3.219

Bijttebier, P., Beck, I., Claes, L., Vandereycken, W., 2009. Gray's Reinforcement Sensitivity Theory as a framework for research on personality-psychopathology associations. Clin. Psychol. Rev. 29, 421-30. doi:10.1016/j.cpr.2009.04.002

Bouton, M.E., 2007. Learning and Behavior. Sinauer Associated, Inc. Publishers, Sunderland, MA.

Bromberg-Martin, E.S., Hikosaka, O., Nakamura, K., 2010a. Coding of task reward value in the dorsal raphe nucleus. J. Neurosci. 30, 6262-6272. doi:10.1523/JNEUROSCI.0015-10.2010

Bromberg-Martin, E.S., Matsumoto, M., Hikosaka, O., 2010b. Dopamine in motivational control: rewarding, aversive, and alerting. Neuron 68, 815-34. doi:10.1016/j.neuron.2010.11.022

Bromberg-Martin, E.S., Matsumoto, M., Nakahara, H., Hikosaka, O., 2010c. Multiple timescales of memory in lateral habenula and dopamine neurons. Neuron 67, 499-510. doi:10.1016/j.neuron.2010.06.031

Bunzeck, N., Guitart-Masip, M., Dolan, R.J., Duzel, E., 2014. Pharmacological dissociation of novelty responses in the human brain. Cereb. Cortex 24, 1351-1360. doi:10.1093/cercor/bhs420

Callan, D.E., Schweighofer, N., 2008. Positive and negative modulation of word learning by reward anticipation. Hum. Brain Mapp. 29, 237-49. doi:10.1002/hbm.20383

Caroni, P., Chowdhury, A., Lahr, M., 2014. Synapse rearrangements upon learning: from divergent-sparse connectivity to dedicated sub-circuits. Trends Neurosci. 37, 604-614. doi:10.1016/j.tins.2014.08.011

Cha, J., Carlson, J.M., Dedora, D.J., Greenberg, T., Proudfit, G.H., Mujica-Parodi, L.R., 2014. Hyperreactive human ventral tegmental area and aberrant mesocorticolimbic connectivity in overgeneralization of fear in generalized anxiety disorder. J. Neurosci. 34, 5855-60. doi:10.1523/JNEUROSCI.4868-13.2014

Chib, V.S., De Martino, B., Shimojo, S., O’Doherty, J.P., 2012. Neural mechanisms underlying paradoxical performance for monetary incentives are driven by loss aversion. Neuron 74, 582-94. doi:10.1016/j.neuron.2012.02.038

Clithero, J. a, Reeck, C., Carter, R.M., Smith, D. V., Huettel, S. a, 2011. Nucleus Accumbens Mediates Relative Motivation for Rewards in the Absence of Choice. Front. Hum. Neurosci. 5, 1-11. doi:10.3389/fnhum.2011.00087 
Cohen, J.D., Perlstein, W.M., Braver, T.S., Nystrom, L.E., Noll, D.C., Jonides, J., Smith, E.E., Cohen et al, J.D., 1997. Temporal dynamics of brain activation during a working memory task. Nature 604-608.

Cohen, M.X., Young, J., Baek, J.-M., Kessler, C., Ranganath, C., 2005. Individual differences in extraversion and dopamine genetics predict neural reward responses. Cogn. Brain Res. 25, 851-61. doi:10.1016/j.cogbrainres.2005.09.018

Cohen, N., Pell, L., Edelson, M.G., Ben-yakov, A., Pine, A., Dudai, Y., 2014. Peri-encoding predictors of memory encoding and consolidation. Neurosci. Biobehav. Rev. doi:10.1016/j.neubiorev.2014.11.002

Colzato, L.S., van den Wildenberg, W.P.M., Hommel, B., 2013. Cognitive control and the COMT Val158Met polymorphism: genetic modulation of videogame training and transfer to taskswitching efficiency. Psychol. Res. 1-9. doi:10.1007/s00426-013-0514-8

Cools, R., Nakamura, K., Daw, N.D., 2011. Serotonin and dopamine: unifying affective, activational, and decision functions. Neuropsychopharmacology 36, 98-113. doi:10.1038/npp.2010.121

Corkin, S., 1968. Acquisition of motor skill after bilateral medial temporal-lobe excision. Neuropsychologia 6, 255-265.

Corr, P.J., 2004. Reinforcement sensitivity theory and personality. Neurosci. Biobehav. Rev. 28, 317-32. doi:10.1016/j.neubiorev.2004.01.005

D’Ardenne, K., McClure, S.M., Nystrom, L.E., Cohen, J.D., 2008. BOLD responses reflecting dopaminergic signals in the human ventral tegmental area. Science 319, 1264-7. doi:10.1126/science.1150605

Daniel, R., Pollmann, S., 2010. Comparing the neural basis of monetary reward and cognitive feedback during information-integration category learning. J. Neurosci. 30, 47-55. doi:10.1523/JNEUROSCI.2205-09.2010

Davachi, L., 2006. Item, context and relational episodic encoding in humans. Curr. Opin. Neurobiol. 16, 693-700. doi:10.1016/j.conb.2006.10.012

Dayan, P., Balleine, B.W., 2002. Reward, Motivation and Reinforcement Learning. Neuron 36, 285-298. doi:10.1016/S0896-6273(02)00963-7

De Martino, B., Fleming, S.M., Garrett, N., Dolan, R.J., 2013. Confidence in value-based choice. Nat. Neurosci. 16, 105-10. doi:10.1038/nn.3279

Deci, E.L., Koestner, R., Ryan, R.M., 1999. A meta-analytic review of experiments examining the effects of extrinsic rewards on intrinsic motivation. Psychol. Bull. 125, 627-700.

Delgado, M.R., Dickerson, K.C., 2012. Reward-Related Learning via Multiple Memory Systems. Biol. Psychiatry 72, 134-41. doi:10.1016/j.biopsych.2012.01.023

Den Ouden, H.E.M., Daw, N.D., Fernandez, G., Elshout, J. a, Rijpkema, M., Hoogman, M., Franke, B., Cools, R., 2013. Dissociable effects of dopamine and serotonin on reversal learning. Neuron 80, 1090-100. doi:10.1016/j.neuron.2013.08.030 
Doya, K., 2002. Metalearning and neuromodulation. Neural Networks 15, 495-506. doi:10.1016/S0893-6080(02)00044-8

Dunsmoor, J.E., Kragel, P. a, Martin, A., Labar, K.S., 2013. Aversive Learning Modulates Cortical Representations of Object Categories. Cereb. cortex 1-14. doi:10.1093/cercor/bht138

Dunsmoor, J.E., Martin, A., LaBar, K.S., 2012. Role of conceptual knowledge in learning and retention of conditioned fear. Biol. Psychol. 89, 300-5. doi:10.1016/j.biopsycho.2011.11.002

Dunsmoor, J.E., Mitroff, S.R., LaBar, K.S., 2009. Generalization of conditioned fear along a dimension of increasing fear intensity. Learn. Mem. 16, 460-9. doi:10.1101/Im.1431609

Eichenbaum, H., 2004. Hippocampus: Cognitive Processes and Neural Representations that Underlie Declarative Memory. Neuron 44, 109-120. doi:10.1016/j.neuron.2004.08.028

El-Amamy, H., Holland, P.C., 2006. Substantia nigra pars compacta is critical to both the acquisition and expression of learned orienting of rats. Eur. J. Neurosci. 24, 270-276. doi:10.1111/j.1460-9568.2006.04896.x

Ellenbogen, J.M., Hu, P.T., Payne, J.D., Titone, D., Walker, M.P., 2007. Human relational memory requires time and sleep. Proc. Natl. Acad. Sci. U. S. A. 104, 7723-8. doi:10.1073/pnas.0700094104

Everitt, B.J., Morris, K.A., O'Brien, A., Robbins, T.W., 1991. The basolateral amygdala-ventral striatal system and conditioned place preference: Further evidence of limbic-striatal interactions underlying reward-related processes. Neuroscience 42, 1-18. doi:10.1016/0306-4522(91)90145-E

Faulkner, P., Deakin, J.F.W., 2014. The role of serotonin in reward, punishment and behavioural inhibition in humans: Insights from studies with acute tryptophan depletion. Neurosci. Biobehav. Rev. 46, 365-378. doi:10.1016/j.neubiorev.2014.07.024

Fenker, D.B., Frey, J.U., Schuetze, H., Heipertz, D., Heinze, H.-J., Duzel, E., 2008. Novel scenes improve recollection and recall of words. J. Cogn. Neurosci. 20, 1250-65. doi:10.1162/jocn.2008.20086

Fields, H.L., Hjelmstad, G.O., Margolis, E.B., Nicola, S.M., 2007. Ventral tegmental area neurons in learned appetitive behavior and positive reinforcement. Annu. Rev. Neurosci. 30, 289-316. doi:10.1146/annurev.neuro.30.051606.094341

Fischer, S., Born, J., 2009. Anticipated reward enhances offline learning during sleep. J. Exp. Psychol. Learn. Mem. Cogn. 35, 1586-93. doi:10.1037/a0017256

Flagel, S.B., Clark, J.J., Robinson, T.E., Mayo, L., Czuj, A., Willuhn, I., Akers, C. a, Clinton, S.M., Phillips, P.E.M., Akil, H., 2011. A selective role for dopamine in stimulus-reward learning. Nature 469, 53-7. doi:10.1038/nature09588

Flannery, M.E., 2011. Cash for Grades? NEA Today Mag. 
Forcato, C., Burgos, V.L., Argibay, P.F., Molina, V.A., Pedreira, M.E., Maldonado, H., 2007. Reconsolidation of declarative memory in humans. Learn. Mem. 14, 295-303. doi:10.1101/Im.486107

Frank, M.J., 2011. Computational models of motivated action selection in corticostriatal circuits. Curr. Opin. Neurobiol. 21, 381-6. doi:10.1016/j.conb.2011.02.013

Frank, M.J., Claus, E.D., 2006. Anatomy of a decision: striato-orbitofrontal interactions in reinforcement learning, decision making, and reversal. Psychol. Rev. 113, 300-326. doi:10.1037/0033-295X.113.2.300

Frank, M.J., O'Reilly, R.C., Curran, T., 2006. When Memory Fails, Intuition Reigns: Midazolam Enhances Implicit Inference in Humans. Psychol. Sci. 17, 700-707. doi:10.1111/j.14679280.2006.01769.x

Franken, I.H.A., 2002. Behavioral approach system (BAS) sensitivity predicts alcohol craving. Pers. Individ. Dif. 32, 349-355. doi:10.1016/S0191-8869(01)00030-7

Franken, I.H.A., Muris, P., Georgieva, I., 2006. Gray's model of personality and addiction. Addict. Behav. 31, 399-403. doi:10.1016/j.addbeh.2005.05.022

Gasbarri, A., Verney, C., Innocenzi, R., Campana, E., Pacitti, C., 1994. Mesolimbic dopaminergic neurons innervating the hippocampal formation in the rat: a combined retrograde tracing and immunohistochemical study. Brain Res. 668, 71-79.

Gheysen, F., Van Opstal, F., Roggeman, C., Van Waelvelde, H., Fias, W., 2011. The neural basis of implicit perceptual sequence learning. Front. Hum. Neurosci. 5, 137. doi:10.3389/fnhum.2011.00137

Ghirlanda, S., Enquist, M., 2003. A century of generalization. Anim. Behav. 66, 15-36. doi:10.1006/anbe.2003.2174

Glimcher, P.W., 2011. Understanding dopamine and reinforcement learning: The dopamine reward prediction error hypothesis. Proc. Natl. Acad. Sci. 108, 15647-15654. doi:10.1073/pnas.1014269108

Gottlieb, J., Hayhoe, M., Hikosaka, O., Rangel, A., 2014. Attention, reward, and information seeking. J. Neurosci. 34, 15497-15504. doi:10.1523/JNEUROSCI.3270-14.2014

Gray, J.A., 1991. The neuropsychology of temperament., in: Strelau, J., Angleitner, A. (Eds.), Explorations in Temperament: International Perspectives on Theory and Measurement. Perspectives on Individual Differences. Plenum Press, York, NY, US, pp. 105-128.

Green, C.S., Bavelier, D., 2008. Exercising Your Brain: A Review of Human Brain Plasticity and Training-Induced Learning. Psychol. Aging 23, 692-701. doi:10.1037/a0014345

Gruber, M.J., Gelman, B.D., Ranganath, C., 2014. States of Curiosity Modulate HippocampusDependent Learning via the Dopaminergic Circuit. Neuron 1-11. doi:10.1016/j.neuron.2014.08.060 
Guitart-Masip, M., Bunzeck, N., Stephan, K.E., Dolan, R.J., Duzel, E., Düzel, E., 2010. Contextual novelty changes reward representations in the striatum. J. Neurosci. 30, 1721-6. doi:10.1523/JNEUROSCI.5331-09.2010

Haber, S.N., Knutson, B., 2010. The reward circuit: linking primate anatomy and human imaging. Neuropsychopharmacology 35, 4-26. doi:10.1038/npp.2009.129

Hahn, T., Dresler, T., Ehlis, A.-C., Plichta, M.M., Heinzel, S., Polak, T., Lesch, K.-P., Breuer, F., Jakob, P.M., Fallgatter, A.J., 2009. Neural response to reward anticipation is modulated by Gray's impulsivity. Neuroimage 46, 1148-53. doi:10.1016/j.neuroimage.2009.03.038

Hall, G., 1996. Learning about associatively activated stimulus representations: Implications for acquired equivalence and perceptual learning 24, 233-255. doi:10.3758/BF03198973

Hickey, C., Chelazzi, L., Theeuwes, J., 2010. Reward changes salience in human vision via the anterior cingulate. J. Neurosci. 30, 11096-103. doi:10.1523/JNEUROSCI.1026-10.2010

Holland, P.C., Gallagher, M., 2003. Double dissociation of the effects of lesions of basolateral and central amygdala on conditioned stimulus-potentiated feeding and Pavlovian-instrumental transfer. Eur. J. Neurosci. 17, 1680-1694.

Holmes, N.M., Marchand, A.R., Coutureau, E., 2010. Pavlovian to instrumental transfer: A neurobehavioural perspective. Neurosci. Biobehav. Rev. 34, 1277-1295. doi:10.1016/j.neubiorev.2010.03.007

Honig, W.K., Urcuioli, P.J., 1981. The legacy of Guttman and Kalish (1956): Twenty-five years of research on stimulus generalization. J. Exp. Anal. Behav. 36, 405-45. doi:10.1901/jeab.1981.36-405

Hoscheidt, S.M., Nadel, L., Payne, J., Ryan, L., 2010. Hippocampal activation during retrieval of spatial context from episodic and semantic memory. Behav. Brain Res. 212, 121-32. doi:10.1016/j.bbr.2010.04.010

Howard-Jones, P., Demetriou, S., Bogacz, R., Yoo, J.H., Leonards, U., 2011. Toward a Science of Learning Games. Mind, Brain Educ. 5, 33-41. doi:10.1111/j.1751-228X.2011.01108.x

Huang, Y., Kandel, E., 1995. D1/D5 Receptor Agonists Induce a Protein Synthesis-Dependent Late Potentiation in the CA1 Region of the Hippocampus. Proc. Natl. Acad. Sci. 92, 2446-2450. doi:10.1073/pnas.92.7.2446

Ikemoto, S., 2007. Dopamine reward circuitry: Two projection systems from the ventral midbrain to the nucleus accumbens-olfactory tubercle complex. Brain Res. Rev. 56, 27-78. doi:10.1016/j.brainresrev.2007.05.004

Jenkins, H.M., Harrison, R.H., 1960. Effect of discrimination training on auditory generalization. J. Exp. Psychol. 59, 246-253. doi:10.1037/h0041661

Johnson, J.D., McDuff, S.G.R., Rugg, M.D., Norman, K. a, 2009. Recollection, familiarity, and cortical reinstatement: a multivoxel pattern analysis. Neuron 63, 697-708. doi:10.1016/j.neuron.2009.08.011 
Jones, J.L., Day, J.J., Aragona, B.J., Wheeler, R.A., Wightman, R.M., Carelli, R.M., 2010. Basolateral amygdala modulates terminal dopamine release in the nucleus accumbens and conditioned responding. Biol. Psychiatry 67, 737-44. doi:10.1016/j.biopsych.2009.11.006

Kahnt, T., Park, S.Q., Burke, C.J., Tobler, P.N., 2012. How glitter relates to gold: similaritydependent reward prediction errors in the human striatum. J. Neurosci. 32, 16521-9. doi:10.1523/JNEUROSCI.2383-12.2012

Kakade, S., Dayan, P., 2002. Dopamine: generalization and bonuses. Neural Networks 15, 549559. doi:10.1016/S0893-6080(02)00048-5

Kang, M.J., Hsu, M., Krajbich, I.M., Loewenstein, G., McClure, S.M., Wang, J.T., Camerer, C.F., 2009. The Wick in the Candle of Learning. Psychol. Sci. 20, 963-974. doi:10.1111/j.14679280.2009.02402.x

Kaplan, F., Oudeyer, P.-Y., 2007. In search of the neural circuits of intrinsic motivation. Front. Neurosci. 1, 225-36. doi:10.3389/neuro.01.1.1.017.2007

Kennedy, P.J., Shapiro, M.L., 2009. Motivational states activate distinct hippocampal representations to guide goal-directed behaviors. Proc. Natl. Acad. Sci. U. S. A. 106, 1080510. doi:10.1073/pnas.0903259106

Knowlton, B.J., Squire, L.R., Gluck, M.A., 1994. Probabilistic classification learning in amnesia. Learn. Mem. 1, 106-120. doi:10.1101/Im.1.2.106

Knutson, B., Adams, C.M., Fong, G.W., Hommer, D., 2001. Anticipation of increasing monetary reward selectively recruits nucleus accumbens., The Journal of neuroscience: the official journal of the Society for Neuroscience. doi:20015472 [pii]

Kranz, G.S., Kasper, S., Lanzenberger, R., 2010. Reward and the serotonergic system. Neuroscience 166, 1023-1035. doi:10.1016/j.neuroscience.2010.01.036

Kravitz, A. V, Tye, L.D., Kreitzer, A.C., 2012. Distinct roles for direct and indirect pathway striatal neurons in reinforcement. Nat. Neurosci. 4-7. doi:10.1038/nn.3100

Krebs, R., Heipertz, D., Schuetze, H., Duzel, E., 2011. Novelty increases the mesolimbic functional connectivity of the substantia nigra/ventral tegmental area (SN/VTA) during reward anticipation: Evidence from high-resolution fMRI. Neuroimage 58, 647-55. doi:10.1016/j.neuroimage.2011.06.038

Krebs, R., Schott, B.H., Düzel, E., 2009. Personality traits are differentially associated with patterns of reward and novelty processing in the human substantia nigra/ventral tegmental area. Biol. Psychiatry 65, 103-10. doi:10.1016/j.biopsych.2008.08.019

Krebs, R.M., Boehler, C.N., Egner, T., Woldorff, M.G., 2011. The neural underpinnings of how reward associations can both guide and misguide attention. J. Neurosci. 31, 9752-9. doi:10.1523/JNEUROSCI.0732-11.2011

Kuhl, B. a, Shah, A.T., DuBrow, S., Wagner, A.D., 2010. Resistance to forgetting associated with hippocampus-mediated reactivation during new learning. Nat. Neurosci. 13, 501-6. doi:10.1038/nn.2498 
Kumaran, D., Summerfield, J.J., Hassabis, D., Maguire, E.A., 2009. Tracking the emergence of conceptual knowledge during human decision making. Neuron 63, 889-901. doi:10.1016/j.neuron.2009.07.030

Lancaster, T.M., Linden, D.E., Heerey, E.A., 2012. COMT val158met predicts reward responsiveness in humans. Genes, Brain Behav. 11, 986-992. doi:10.1111/j.1601183X.2012.00838.x

Lansink, C.S., Goltstein, P.M., Lankelma, J. V, McNaughton, B.L., Pennartz, C.M.A., 2009. Hippocampus leads ventral striatum in replay of place-reward information. PLoS Biol. 7, e1000173. doi:10.1371/journal.pbio.1000173

Lee, H.J., Youn, J.M., O, M.J., Gallagher, M., Holland, P.C., 2006. Role of Substantia NigraAmygdala Connections in Surprise-Induced Enhancement of Attention. J. Neurosci. 26 , 6077-6081. doi:10.1523/JNEUROSCI.1316-06.2006

Lewis, P. a, Durrant, S.J., 2011. Overlapping memory replay during sleep builds cognitive schemata. Trends Cogn. Sci. 15, 343-51. doi:10.1016/j.tics.2011.06.004

Liljeholm, M., O'Doherty, J.P., 2012. Contributions of the striatum to learning, motivation, and performance: an associative account. Trends Cogn. Sci. 16, 467-75. doi:10.1016/j.tics.2012.07.007

Linke, J., Kirsch, P., King, A. V, Gass, A., Hennerici, M.G., Bongers, A., Wessa, M., 2010. Motivational orientation modulates the neural response to reward. Neuroimage 49, 261825. doi:10.1016/j.neuroimage.2009.09.013

Lisman, J., Grace, A., 2005. The hippocampal-VTA loop: controlling the entry of information into long-term memory. Neuron 46, 703-713. doi:10.1016/j.neuron.2005.05.002

Lisman, J., Grace, A., Duzel, E., 2011. A neoHebbian framework for episodic memory; role of dopamine-dependent late LTP. Trends Neurosci. 34, 536-47. doi:10.1016/j.tins.2011.07.006

Luo, A.H., Tahsili-Fahadan, P., Wise, R. a, Lupica, C.R., Aston-Jones, G., 2011. Linking Context with Reward: A Functional Circuit from Hippocampal CA3 to Ventral Tegmental Area. Science (80. ). 353, 353-7. doi:10.1126/science.1204622

Madan, C.R., Fujiwara, E., Gerson, B.C., Caplan, J.B., 2012. High reward makes items easier to remember, but harder to bind to a new temporal context. Front. Integr. Neurosci. 6, Article 61. doi:10.3389/fnint.2012.00061

Mahler, S. V, Berridge, K.C., 2009. Which Cue to "Want?" Central Amygdala Opioid Activation Enhances and Focuses Incentive Salience on a Prepotent Reward Cue. J. Neurosci. 29, 65006513. doi:10.1523/JNEUROSCI.3875-08.2009

Mahler, S. V., Berridge, K.C., 2012. What and when to "want"? Amygdala-based focusing of incentive salience upon sugar and sex. Psychopharmacology (Berl). 221, 407-426. doi:10.1007/s00213-011-2588-6

Maren, S., Phan, K.L., Liberzon, I., 2013. The contextual brain: implications for fear conditioning, extinction and psychopathology. Nat. Rev. Neurosci. 14, 417-28. doi:10.1038/nrn3492 
Martin, L.E., Potts, G.F., 2004. Reward sensitivity in impulsivity. Neuroreport 15, 1519-1522. doi:10.1097/01.wnr.0000132920.12990.b9

Martin-Soelch, C., Linthicum, J., Ernst, M., 2007. Appetitive conditioning: neural bases and implications for psychopathology. Neurosci. Biobehav. Rev. 31, 426-40. doi:10.1016/j.neubiorev.2006.11.002

Mather, M., Harley, C.W., Clewett, D., Sakaki, M., Harley, C.W., 2015. Norepinephrine ignites local hot spots of neuronal excitation: How arousal amplifies selectivity in perception and memory. Behav. Brain Sci. 1, 1-100.

Mather, M., Schoeke, A., 2011. Positive outcomes enhance incidental learning for both younger and older adults. Front. Neurosci. 5, 129. doi:10.3389/fnins.2011.00129

Mather, M., Sutherland, M.R., 2011. Arousal-biased competition in perception and memory. Perspect. Psychol. Sci. 6, 114-133. doi:10.1177/1745691611400234

McAuley, E., Duncan, T., Tammen, V., 1989. Psychometric properties of the intrinsic motivation inventory in a competitive sport setting: a confirmatory factor analysis. Res. Q. Exerc. Sport 48-58. doi:10.1080/02701367.1989.10607413

McKenzie, S., Eichenbaum, H., 2011. Consolidation and reconsolidation: two lives of memories? Neuron 71, 224-33. doi:10.1016/j.neuron.2011.06.037

Mcnamara, C.G., Tejero-cantero, Á., Trouche, S., Campo-urriza, N., Dupret, D., 2014. Dopaminergic neurons promote hippocampal reactivation and spatial memory persistence. Nat. Neurosci. 17, 1658-1660. doi:10.1038/nn.3843

Mendelsohn, D., Riedel, W.J., Sambeth, A., 2009. Effects of acute tryptophan depletion on memory, attention and executive functions: A systematic review. Neurosci. Biobehav. Rev. 33, 926-952. doi:10.1016/j.neubiorev.2009.03.006

Meyer, P.J., Lovic, V., Saunders, B.T., Yager, L.M., Flagel, S.B., Morrow, J.D., Robinson, T.E., 2012. Quantifying individual variation in the propensity to attribute incentive salience to reward cues. PLoS One 7, e38987. doi:10.1371/journal.pone.0038987

Mizuno, K., Tanaka, M., Ishii, A., Tanabe, H.C., Onoe, H., Sadato, N., Watanabe, Y., 2008. The neural basis of academic achievement motivation. Neuroimage 42, 369-78. doi:10.1016/j.neuroimage.2008.04.253

Mobbs, D., Hassabis, D., Seymour, B., Marchant, J.L., Weiskopf, N., Dolan, R.J., Frith, C.D., 2009. Choking on the Money. Reward-Based Performance Decrements Are Associated With Midbrain Activity. Psychol. Sci. 20, 955-963. doi:10.1111/j.1467-9280.2009.02399.x

Morris, J.S., Dolan, R.J., 2001. Involvement of human amygdala and orbitofrontal cortex in hungerenhanced memory for food stimuli. J. Neurosci. 21, 5304-10.

Morrison, S.E., Salzman, C.D., 2010. Re-valuing the amygdala. Curr. Opin. Neurobiol. 20, 221-30. doi:10.1016/j.conb.2010.02.007 
Moses, S.N., Brown, T.M., Ryan, J.D., McIntosh, A.R., 2010. Neural system interactions underlying human transitive inference. Hippocampus 20, 894-901. doi:10.1002/hipo.20735

Murayama, K., Kuhbandner, C., 2011. Money enhances memory consolidation--but only for boring material. Cognition 119, 120-4. doi:10.1016/j.cognition.2011.01.001

Murayama, K., Matsumoto, M., Izuma, K., Matsumoto, K., 2010. Neural basis of the undermining effect of monetary reward on intrinsic motivation. Proc. Natl. Acad. Sci. U. S. A. 107, 209116. doi:10.1073/pnas.1013305107

Murty, V.P., Adcock, R.A., 2014. Enriched Encoding: Reward Motivation Organizes Cortical Networks for Hippocampal Detection of Unexpected Events. Cereb. Cortex 24, 2160'2168. doi:10.1093/cercor/bht063

Myers, C.E., Shohamy, D., Gluck, M.A., Grossman, S., Kluger, A., Ferris, S., Golomb, J., Schnirman, G., Schwartz, R., 2003. Dissociating Hippocampal versus Basal Ganglia Contributions to Learning and Transfer. J. Cogn. Neurosci. 15, 185-193. doi:10.1162/089892903321208123

Nadel, L., Hupbach, a., Gomez, R., Newman-Smith, K., 2012. Memory formation, consolidation and transformation. Neurosci. Biobehav. Rev. 36, 1640-1645. doi:10.1016/j.neubiorev.2012.03.001

Nadel, L., Moscovitch, M., 1997. Memory consolidation, retrograde amnesia and the hippocampal complex. Curr. Opin. Neurobiol. 7, 217-27. doi:10.1016/S0959-4388(97)80010-4

Niv, Y., Daw, N.D., Joel, D., Dayan, P., 2007. Tonic dopamine: opportunity costs and the control of response vigor. Psychopharmacology (Berl). 191, 507-20. doi:10.1007/s00213-006-0502-4

O'Carroll, C.M., Martin, S.J., Sandin, J., Frenguelli, B., Morris, R.G.M., 2006. Dopaminergic modulation of the persistence of one-trial hippocampus-dependent memory. Learn. Mem. 13, 760-769. doi:10.1101/Im.321006

Olshavsky, M.E., Song, B.J., Powell, D.J., Jones, C.E., Monfils, M.-H., Lee, H.J., 2013. Updating appetitive memory during reconsolidation window: critical role of cue-directed behavior and amygdala central nucleus. Front. Behav. Neurosci. 7, 186. doi:10.3389/fnbeh.2013.00186

Oudiette, D., Antony, J.W., Creery, J.D., Paller, K.A., 2013. The role of memory reactivation during wakefulness and sleep in determining which memories endure. J. Neurosci. 33, 6672-8. doi:10.1523/JNEUROSCI.5497-12.2013

Packard, M.G., Goodman, J., 2012. Emotional arousal and multiple memory systems in the mammalian brain. Front. Behav. Neurosci. 6, 14. doi:10.3389/fnbeh.2012.00014

Paton, J.J., Belova, M.A., Morrison, S.E., Salzman, C.D., 2006. The primate amygdala represents the positive and negative value of visual stimuli during learning. Nature 439, 865-870.

Pavlov, I.P., 1927. Conditioned reflexes: an investigation of the physiological activity of the cerebral cortex. Oxford University Press. 
Pecina, S., Cagniard, B., Berridge, K.C., Aldridge, J.W., Zhuang, X., 2003. Hyperdopaminergic mutant mice have higher "wanting" but not "liking" for sweet rewards. J. Neurosci. 23, 9395-9402.

Pennartz, C., Ito, R., Verschure, P., Battaglia, F., Robbins, T., 2011. The hippocampal-striatal axis in learning, prediction and goal-directed behavior. Trends Neurosci. 34, 548-59. doi:10.1016/j.tins.2011.08.001

Pennartz, C.M.A., Berke, J., Graybiel, A., Ito, R., Lansink, C., van der Meer, M., Redish, A.D., Smith, K.S., Voorn, P., 2009. Corticostriatal Interactions during Learning, Memory Processing, and Decision Making. J. Neurosci. 29, 12831-8. doi:10.1523/jneurosci.3177-09.2009

Perogamvros, L., Schwartz, S., 2012. The roles of the reward system in sleep and dreaming. Neurosci. Biobehav. Rev. 36, 1934-51. doi:10.1016/j.neubiorev.2012.05.010

Poldrack, R.A., Foerde, K., 2008. Category learning and the memory systems debate. Neurosci. Biobehav. Rev. 32, 197-205. doi:10.1016/j.neubiorev.2007.07.007

Preston, A.R., Eichenbaum, H., 2013. Interplay of hippocampus and prefrontal cortex in memory. Curr. Biol. 23, R764-73. doi:10.1016/j.cub.2013.05.041

Rasch, B., Born, J., 2013. About sleep's role in memory. Physiol. Rev. 93, 681-766. doi:10.1152/physrev.00032.2012

Raymond, J.E., O'Brien, J.L., 2009. Selective Visual Attention and Motivation. Psychol. Sci. 20, 981988. doi:10.1111/j.1467-9280.2009.02391.x

Robbins, T.W., Everitt, B.J., 2007. A role for mesencephalic dopamine in activation: commentary on Berridge (2006). Psychopharmacology (Berl). 191, 433-7. doi:10.1007/s00213-006-05287

Roelfsema, P.R., van Ooyen, A., Watanabe, T., 2010. Perceptual learning rules based on reinforcers and attention. Trends Cogn. Sci. 14, 64-71. doi:10.1016/j.tics.2009.11.005

Roozendaal, B., McGaugh, J.L., 2011. Memory modulation. Behav. Neurosci. 125, 797-824. doi:10.1037/a0026187

Rose, M., Haider, H., Büchel, C., 2010. The emergence of explicit memory during learning. Cereb. Cortex 20, 2787-2797. doi:10.1093/cercor/bhq025

Rose, M., Haider, H., Salari, N., Büchel, C., 2011. Functional dissociation of hippocampal mechanism during implicit learning based on the domain of associations. J. Neurosci. 31, 13739-45. doi:10.1523/JNEUROSCI.3020-11.2011

Rossato, J., Bevilaqua, L., Izquierdo, I., Medina, J., Cammarota, M., 2009. Dopamine Controls Persistence of Long-Term Memory Storage. Science (80-. ). 325, 1017-1020. doi:10.1126/science.1172545

Rushworth, M., Noonan, M., Boorman, E., Walton, M., Behrens, T.E., 2011. Frontal cortex and reward-guided learning and decision-making. Neuron 70, 1054-69. doi:10.1016/j.neuron.2011.05.014 
Ryan, R., Deci, E., 2000. Intrinsic and Extrinsic Motivations: Classic Definitions and New Directions. Contemp. Educ. Psychol. 25, 54-67. doi:10.1006/ceps.1999.1020

Rygula, R., Clarke, H.F., Cardinal, R.N., Cockcroft, G.J., Xia, J., Dalley, J.W., Robbins, T.W., Roberts, A.C., 2014. Role of Central Serotonin in Anticipation of Rewarding and Punishing Outcomes: Effects of Selective Amygdala or Orbitofrontal 5-HT Depletion. Cereb. Cortex bhu102. doi:10.1093/cercor/bhu102

Sadeh, T., Shohamy, D., Levy, D.R., Reggev, N., Maril, A., 2011. Cooperation between the hippocampus and the striatum during episodic encoding. J. Cogn. Neurosci. 23, 1597-608. doi:10.1162/jocn.2010.21549

Salamone, J.D., Correa, M., 2012. The Mysterious Motivational Functions of Mesolimbic Dopamine. Neuron 76, 470-485. doi:10.1016/j.neuron.2012.10.021

Salimpoor, V.N., Benovoy, M., Larcher, K., Dagher, A., Zatorre, R.J., 2011. Anatomically distinct dopamine release during anticipation and experience of peak emotion to music. Nat. Neurosci. 14, 257-262. doi:10.1038/nn.2726

Salvetti, B., Morris, R.G.M., Wang, S., 2014. The role of rewarding and novel events in facilitating memory persistence in a separate spatial memory task. Learn. Mem. 61-72.

Sara, S.J., 2009. The locus coeruleus and noradrenergic modulation of cognition. Nat. Rev. Neurosci. 10, 211-23. doi:10.1038/nrn2573

Sasaki, Y., Nanez, J.E., Watanabe, T., 2010. Advances in visual perceptual learning and plasticity. Nat. Rev. Neurosci. 11, 53-60. doi:10.1038/nrn2737

Schacter, D., Gilbert, D., Wegner, D., 2010. Psychology, 2nd ed. Worth Publishers.

Schechtman, E., Laufer, O., Paz, R., 2010. Negative Valence Widens Generalization of Learning. J. Neurosci. 30, 10460-10464. doi:10.1523/JNEUROSCI.2377-10.2010

Schomaker, J., Meeter, M., 2015. Short- and long-lasting consequences of novelty, deviance and surprise on brain and cognition. Neurosci. Biobehav. Rev. 55, 268-279. doi:10.1016/j.neubiorev.2015.05.002

Schomaker, J., van Bronkhorst, M.L.V., Meeter, M., 2014. Exploring a novel environment improves motivation and promotes recall of words. Front. Psychol. 5, 1-6. doi:10.3389/fpsyg.2014.00918

Schott, B.H., Seidenbecher, C.I., Fenker, D.B., Lauer, C.J., Bunzeck, N., Bernstein, H.-G., Tischmeyer, W., Gundelfinger, E.D., Heinze, H.-J., Düzel, E., 2006. The dopaminergic midbrain participates in human episodic memory formation: evidence from genetic imaging. J. Neurosci. 26, 1407-1417. doi:10.1523/JNEUROSCI.3463-05.2006

Schott, B.H., Sellner, D.B., Lauer, C.J., Habib, R., Frey, J.U., Guderian, S., Heinze, H.J., Düzel, E., 2004. Activation of midbrain structures by associative novelty and the formation of explicit memory in humans. Learn. Mem. 11, 383-387. doi:10.1101/lm.75004 
Schultz, W., 2007. Multiple dopamine functions at different time courses. Annu. Rev. Neurosci. 30, 259-88. doi:10.1146/annurev.neuro.28.061604.135722

Schultz, W., 2010. Dopamine signals for reward value and risk: basic and recent data. Behav. Brain Funct. 6, 24. doi:10.1186/1744-9081-6-24

Schultz, W., Preuschoff, K., Camerer, C., Hsu, M., Fiorillo, C.D., Tobler, P.N., Bossaerts, P., 2008. Explicit neural signals reflecting reward uncertainty. Philos. Trans. R. Soc. Lond. B. Biol. Sci. 363, 3801-11. doi:10.1098/rstb.2008.0152

Schwabe, L., Wolf, O.T., 2013. Stress and multiple memory systems: from "thinking" to "doing". Trends Cogn. Sci. 17, 60-8. doi:10.1016/j.tics.2012.12.001

Schweighofer, N., Bertin, M., Shishida, K., Okamoto, Y., Tanaka, S.C., Yamawaki, S., Doya, K., 2008. Low-serotonin levels increase delayed reward discounting in humans. J. Neurosci. 28, 45284532. doi:10.1523/JNEUROSCI.4982-07.2008

Scimeca, J.M., Badre, D., 2012. Striatal contributions to declarative memory retrieval. Neuron 75, 380-92. doi:10.1016/j.neuron.2012.07.014

Seger, C.A., Dennison, C.S., Lopez-Paniagua, D., Peterson, E.J., Roark, A. a, 2011. Dissociating hippocampal and basal ganglia contributions to category learning using stimulus novelty and subjective judgments. Neuroimage 55, 1739-53. doi:10.1016/j.neuroimage.2011.01.026

Seitz, A., Watanabe, T., 2005. A unified model for perceptual learning. Trends Cogn. Sci. 9, 329334. doi:10.1016/j.tics.2005.05.010

Seyedabadi, M., Fakhfouri, G., Ramezani, V., Mehr, S.E., Rahimian, R., 2014. The role of serotonin in memory: interactions with neurotransmitters and downstream signaling. Exp. Brain Res. 1-16. doi:10.1007/s00221-013-3818-4

Sharot, T., Guitart-Masip, M., Korn, C.W., Chowdhury, R., Dolan, R.J., 2012. How dopamine enhances an optimism bias in humans. Curr. Biol. 22, 1477-81. doi:10.1016/j.cub.2012.05.053

Shibata, K., Yamagishi, N., Ishii, S., Kawato, M., 2009. Boosting perceptual learning by fake feedback. Vision Res. 49, 2574-2585.

Shohamy, D., Adcock, R.A., 2010. Dopamine and adaptive memory. Trends Cogn. Sci. 14, 464-72. doi:10.1016/j.tics.2010.08.002

Shohamy, D., Myers, C.E., Kalanithi, J., Gluck, M. a, 2008. Basal ganglia and dopamine contributions to probabilistic category learning. Neurosci. Biobehav. Rev. 32, 219-36. doi:10.1016/j.neubiorev.2007.07.008

Shohamy, D., Wagner, A.D., 2008. Integrating memories in the human brain: hippocampalmidbrain encoding of overlapping events. Neuron 60, 378-89. doi:10.1016/j.neuron.2008.09.023 
Simon, J.J., Walther, S., Fiebach, C.J., Friederich, H.-C., Stippich, C., Weisbrod, M., Kaiser, S., 2010. Neural reward processing is modulated by approach- and avoidance-related personality traits. Neuroimage 49, 1868-74. doi:10.1016/j.neuroimage.2009.09.016

Singer, A., Frank, L., 2009. Rewarded outcomes enhance reactivation of experience in the hippocampus. Neuron 64, 910-21. doi:10.1016/j.neuron.2009.11.016

Singer, T., Critchley, H.D., Preuschoff, K., 2009. A common role of insula in feelings, empathy and uncertainty. Trends Cogn. Sci. 13, 334-40. doi:10.1016/j.tics.2009.05.001

Smith, C.C., Greene, R.W., 2012. CNS dopamine transmission mediated by noradrenergic innervation. J. Neurosci. 32, 6072-6080.

Smith, K.S., Graybiel, A.M., 2013. A Dual Operator View of Habitual Behavior Reflecting Cortical $\begin{array}{llll}\text { and Striatal Neuron } \quad \text { Dynamics. } & \text { 361-374. }\end{array}$ doi:http://dx.doi.org/10.1016/j.neuron.2013.05.038

Smith, W.B., Starck, S.R., Roberts, R.W., Schuman, E.M., 2005. Dopaminergic stimulation of local protein synthesis enhances surface expression of GluR1 and synaptic transmission in hippocampal neurons. Neuron 45, 765-779.

Squire, L.R., 1992. Memory and the hippocampus: a synthesis from findings with rats, monkeys, and humans. Psychol. Rev. 99, 195-231.

Squire, L.R., Wixted, J.T., 2011. The cognitive neuroscience of human memory since H.M. Annu. Rev. Neurosci. 34, 259-88. doi:10.1146/annurev-neuro-061010-113720

Squire, L.R., Wixted, J.T., Clark, R.E., 2007. Recognition memory and the medial temporal lobe: a new perspective. Nat. Rev. Neurosci. 8, 872-883. doi:10.1038/nrn2154

Steinberg, E.E., Keiflin, R., Boivin, J.R., Witten, I.B., Deisseroth, K., Janak, P.H., 2013. A causal link between prediction errors, dopamine neurons and learning. Nat Neurosci 16, 966-973. doi:10.1038/nn.3413

Sterpenich, V., D’Argembeau, A., Desseilles, M., Balteau, E., Albouy, G., Vandewalle, G., Degueldre, C., Luxen, A., Collette, F., Maquet, P., D’Argembeau, A., Desseilles, M., Balteau, E., Albouy, G., Vandewalle, G., Degueldre, C., Luxen, A., Collette, F., Maquet, P., 2006. The Locus Ceruleus Is Involved in the Successful Retrieval of Emotional Memories in Humans. J. Neurosci. 26 , 7416-7423. doi:10.1523/JNEUROSCI.1001-06.2006

Stickgold, R., Walker, M.P., 2013. Sleep-dependent memory triage: evolving generalization through selective processing. Nat. Neurosci. 16, 139-45. doi:10.1038/nn.3303

Takahashi, H., Yamada, M., Suhara, T., 2012. Functional significance of central D1 receptors in cognition: beyond working memory. J. Cereb. Blood Flow Metab. 32, 1248-58. doi:10.1038/jcbfm.2011.194

Talmi, D., 2013. Enhanced Emotional Memory: Cognitive and Neural Mechanisms. Curr. Dir. Psychol. Sci. 22, 430-436. doi:10.1177/0963721413498893 
Talmi, D., Seymour, B., Dayan, P., Dolan, R.J., 2008. Human pavlovian-instrumental transfer. J. Neurosci. 28, 360-8. doi:10.1523/JNEUROSCI.4028-07.2008

Thomas, S. a., 2015. Neuromodulatory signaling in hippocampus-dependent memory retrieval. Hippocampus 25, 415-431. doi:10.1002/hipo.22394

Tobler, P.N., O'Doherty, J.P., Dolan, R.J., Schultz, W., 2007. Reward value coding distinct from risk attitude-related uncertainty coding in human reward systems., Journal of neurophysiology. Am Physiological Soc. doi:10.1152/jn.00745.2006

Tully, K., Bolshakov, V.Y., 2010. Emotional enhancement of memory: how norepinephrine enables synaptic plasticity. Mol. Brain 3, 15. doi:10.1186/1756-6606-3-15

Tulving, E., 1972. Episodic and Semantic Memory. Organ. Mem. 381-402.

Van den Bos, R., van der Harst, J., Vijftigschild, N., Spruijt, B., van Luijtelaar, G., Maes, R., 2004. On the relationship between anticipatory behaviour in a Pavlovian paradigm and Pavlovian-toInstrumental Transfer in rats (Rattus norvegicus). Behav. Brain Res. 153, 397-408. doi:10.1016/j.bbr.2003.12.017

Van Kesteren, M.T.R., Fernández, G., Norris, D.G., Hermans, E.J., 2010. Persistent schemadependent hippocampal-neocortical connectivity during memory encoding and postencoding rest in humans. Proc. Natl. Acad. Sci. U. S. A. 107, 7550-5. doi:10.1073/pnas.0914892107

Van Kesteren, M.T.R., Ruiter, D.J., Fernández, G., Henson, R.N., 2012. How schema and novelty augment memory formation. Trends Neurosci. 35, 211-9. doi:10.1016/j.tins.2012.02.001

Varazzani, C., San-Galli, a., Gilardeau, S., Bouret, S., 2015. Noradrenaline and Dopamine Neurons in the Reward/Effort Trade-Off: A Direct Electrophysiological Comparison in Behaving Monkeys. J. Neurosci. 35, 7866-7877. doi:10.1523/JNEUROSCI.0454-15.2015

Vervliet, B., Iberico, C., Vervoort, E., Baeyens, F., 2011. Generalization gradients in human predictive learning: Effects of discrimination training and within-subjects testing. Learn. Motiv. 42, 210-220. doi:10.1016/j.Imot.2011.03.004

Vuilleumier, P., Driver, J., 2007. Modulation of visual processing by attention and emotion: windows on causal interactions between human brain regions. Philos. Trans. R. Soc. 362, 837-855. doi:10.1098/rstb.2007.2092

Wächter, T., Lungu, O. V, Liu, T., Willingham, D.T., Ashe, J., 2009. Differential effect of reward and punishment on procedural learning. J. Neurosci. 29, 436-443. doi:10.1523/JNEUROSCI.413208.2009

Wagner, U., Gais, S., Haider, H., Verleger, R., Born, J., 2004. Sleep inspires insight. Nature 427, 352-5. doi:10.1038/nature02223

Wang, S.-H., Morris, R.G.M., 2010. Hippocampal-neocortical interactions in memory formation, consolidation, and reconsolidation. Annu. Rev. Psychol. 61, 49-79, C1-4. doi:10.1146/annurev.psych.093008.100523 
Wang, S.-H., Redondo, R.L., Morris, R.G.M., 2010. Relevance of synaptic tagging and capture to the persistence of long-term potentiation and everyday spatial memory. Proc. Natl. Acad. Sci. U. S. A. 107, 19537-42. doi:10.1073/pnas.1008638107

Werlen, E., Jones, M.W., 2015. Modulating the map: dopaminergic tuning of hippocampal spatial coding and interactions, 1st ed, The Connected Hippocampus. Elsevier B.V. doi:10.1016/bs.pbr.2015.03.002

Wickens, J.R., Reynolds, J.N.J., Hyland, B.I., 2003. Neural mechanisms of reward-related motor learning. Curr. Opin. Neurobiol. 13, 685-690. doi:http://dx.doi.org/10.1016/j.conb.2003.10.013

Wilhelm, I., Diekelmann, S., Molzow, I., Ayoub, A., Mölle, M., Born, J., 2011. Sleep selectively enhances memory expected to be of future relevance. J. Neurosci. 31, 1563-9. doi:10.1523/JNEUROSCI.3575-10.2011

Wimber, M., Schott, B.H., Wendler, F., Seidenbecher, C.I., Behnisch, G., Macharadze, T., Bäuml, K.H.T., Richardson-Klavehn, A., 2011. Prefrontal dopamine and the dynamic control of human long-term memory. Transl. Psychiatry 1, e15. doi:10.1038/tp.2011.15

Wimmer, G.E., Shohamy, D., 2011. The striatum and beyond: Hippocampal contributions to decision making. Atten. Perform. XXII 281-309.

Wimmer, G.E., Shohamy, D., 2012. Preference by association: how memory mechanisms in the hippocampus bias decisions. Science (80-. ). 338, 270-3. doi:10.1126/science.1223252

Wimmer, G.E.E., Daw, N.D., Shohamy, D., 2012. Generalization of value in reinforcement learning by humans. Eur. J. Neurosci. 35, 1092-1104. doi:10.1111/j.1460-9568.2012.08017.x

Wimmer, X.G.E., Braun, E.K., Daw, N.D., Shohamy, D., 2014. Episodic Memory Encoding Interferes with Reward Learning and Decreases Striatal Prediction Errors. J. Neurosci. 34, 1490114912. doi:10.1523/JNEUROSCI.0204-14.2014

Wittmann, B.C., Dolan, R.J., Du, E., 2011. Behavioral specifications of reward-associated long-term memory enhancement in humans. Learn. Mem. 296 -300. doi:10.1101/Im.1996811

Wittmann, B.C., Schott, B.H., Guderian, S., Frey, J.U., Heinze, H.-J., Düzel, E., 2005. Reward-related FMRI activation of dopaminergic midbrain is associated with enhanced hippocampusdependent long-term memory formation. Neuron 45, 459-67. doi:10.1016/j.neuron.2005.01.010

Wolosin, S.M., Zeithamova, D., Preston, A.R., 2012. Reward Modulation of Hippocampal Subfield Activation during Successful Associative Encoding and Retrieval. J. Cogn. Neurosci. 24, 153247. doi:10.1162/jocn_a_00237

Yasuda, M., Yamamoto, S., Hikosaka, O., 2012. Robust representation of stable object values in the oculomotor Basal Ganglia. J. Neurosci. 32, 16917-32. doi:10.1523/JNEUROSCl.343812.2012

Zalesak, M., Heckers, S., 2009. The role of the hippocampus in transitive inference. Psychiatry Res. 172, 24-30. doi:10.1016/j.pscychresns.2008.09.008 
Zeithamova, D., Schlichting, M.L., Preston, A.R., 2012. The hippocampus and inferential reasoning: building memories to navigate future decisions. Front. Hum. Neurosci. 6, 1-14. doi:10.3389/fnhum.2012.00070

Zweynert, S., Pade, J.P., Wüstenberg, T., Sterzer, P., Walter, H., Seidenbecher, C.I., RichardsonKlavehn, A., Düzel, E., Schott, B.H., 2011. Motivational salience modulates hippocampal repetition suppression and functional connectivity in humans. Front. Hum. Neurosci. 5, 144. doi:10.3389/fnhum.2011.00144 


\section{Figure Captions}

Figure 1 Schema of anatomical brain substrates involved in memory modulation by reward.

A. Functional roles of brain substrates as found primarily in animal studies. Memory enhancement is produced by dopaminergic activity projecting from the SN/VTA to the hippocampus (the upward arc), where dopamine has been shown to modulate plasticity (Bromberg-Martin et al., 2010b). The downward arc of the hippocampal-VTA loop, extending from the hippocampus to the subiculum, the nucleus accumbens and pallidum, down to the VTA, is activated by motivational importance of novel information (Lisman and Grace, 2005). In addition to the downward arc, there is an indirect projection from the hippocampus to the VTA (Luo et al., 2011). The integration of inputs to the VTA takes place in the ventral striatum. This input combines information about current motivational goals and task set from the PFC (mPFC in rats, vmPFC in humans, see Bromberg-Martin, Matsumoto \& Hikosaka 2010 as well as Rushworth, Noonan, Boorman et al., 2011 about the role of PFC), sensory stimulus properties from BLA / hippocampus, and the context of the memory from the hippocampus (Pennartz, Ito, Verschure, Battaglia, \& Robbins, 2011), and serves to either promote or inhibit goal-directed behaviors (Schultz, 2010). PFC participates in memory consolidation by assimilating new information with existing mental schemas (Preston and Eichenbaum, 2013), while emotional arousal can bias memory processing via connections between the amygdala and the hippocampus. The amygdala facilitates responding to Pavlovian stimuli by modulating dopamine signals within the core of the nucleus accumbens (Everitt et al., 1991; Jones et al., 2010; Mahler and Berridge, 2009). It is also implicated in switching between goal-directed learning mediated by the MTL and goal-independent habit learning in the basal ganglia. Abbreviations: SN/VTA - substantia nigra/ventral tegmental area; vmPFC - ventro-medial prefrontal cortex; BLA - baso-lateral amygdala; MTL - medial temporal lobe.

B. Regions from A displayed on a human brain anatomical representation 
Figure 2 Substrates of memory modulation by reward.

A Coactivation of midbrain and hippocampus during encoding of rewarded items leads to better memory (green, blue and violet) and associative inference (turquoise).

B Hippocampal-striatal coactivation in generalization of reward value.

Figure 3 Transfer of learned reward value: biases in perception and behavior

A. Generalization of conditioned response to stimuli perceptually similar to the rewardconditioned ones. Gabor patches of 2 different orientations were presented to participants in a Pavlovian conditioning (training phase) with monetary reward (CS+ and CS-). In a subsequent test phase, gratings of orientations differing from the two previously presented were shown to the participants with the task to judge whether the grating predicted reward or not. The responses of the participants are illustrated in the generalization response gradient (right side of the panel) which shows that the reward value generalizes over several orientations close to that of the CS+, with a characteristic peak in reward probability estimation occurring to the left of the orientation of actual CS+ and away from the orientation of the actual CS- (i.e., the "peak shift"; Kahnt et al., 2012).

B. Reward value can generalize via association. During the association phase, participants memorized images of body parts (S1) paired with images of fractals (S2). During a subsequent reward phase, half of the fractals were associated with monetary reward (CS+) via Pavlovian conditioning. Finally, in the decision phase of the experiment, participants were asked to judge which of the two presented pictures (both S1 or both S2) was more likely to predict the monetary reward. During this phase, many participants preferred those S1 (S1+) images whose counterpart S2 (S2+) had been paired with reward. This bias correlated with activation in the posterior hippocampus during the reward phase, which in turn was functionally related to activity in the caudate during the same reward phase (Wimmer and Shohamy, 2012). 
C. Reward value can transfer to a subsequent instrumental task and increase response vigor even when it does not lead to reward. In the first phase of the experiment, participants are reward conditioned to a specific visual background and a sound that signals monetary reward (CS+). Two other background and sound contexts were presented as CS- and a control condition. Next, they were trained to squeeze a grip in order to obtain the same reward. The immediately subsequent third stage, a test for Pavlovian to Instrumental Transfer was conducted in the absence of monetary rewards. All three stimuli sets from phase 1 were shown and the participant was allowed to continue instrumental responding as in phase 2 . There were more hand grips when the CS+ background was on the screen as compared to the CS- or neutral background image, demonstrating Pavlovian-to-instrumental transfer. (Talmi et al., 2008) .

All figures reproduced with permission.

Figure 4 Reward value transfer: enhancement of declarative memory

A. Expectancy violation task with rewarded blocks illustrates the penumbra hypothesis: in a series of identical color images, the participants awaited a grayscale target image the response to which was rewarded with the previously cued amount. The oddball pictures that appeared in high reward anticipation trials were remembered better, as revealed in a recognition memory task administered at the end of the experiment (Murty and Adcock, 2014)

B. Two phases of an associative inference paradigm. Participants first learned a series of facescene associations based on feedback (which can act as an internal reward signal, see section 2). There were three learning event types in which the individual associations shared overlapping features, with two faces associated with a common scene, and one of those faces also associated with a second scene. In a subsequent test phase, participants were asked to respond to untrained face-scene associations. No feedback was provided. The test revealed 
that new (untrained) associations had been formed during learning due to integrative encoding triggered by the overlapping features across individual trained associations. (Shohamy and Wagner, 2008)

C. This subsequent associative learning paradigm can induce retroactive interference whereby $A C$ pairs (Interference) overwrite a previously learned $A B$ association (Encoding). When $A B$ had been paired with high reward, activation in the hippocampus as well as reward-related areas (vmPFC and ventral striatum) during AC encoding correlated with the strength of the subsequently tested $A B$ memory (i.e. its protection from forgetting due to interference), an effect that may involve the reactivation and consolidation of the initially rewarded $A B$ pair (Kuhl et al., 2010).

All figures reproduced with permission. 


\section{Box 1. Terminology}

Declarative memory: memories that can be declared; that is, they have a propositional truth value (events or facts) and are normally associated with conscious recall. They are distinct from procedural (non-declarative) memories such as skill learning, which cannot be verbalized and are often expressed unconsciously (van Kesteren et al., 2012).

Generalization vs Transfer. In the perceptual and skill learning domain, generalization refers to the process leading to the ability to recognize commonalities between what was previously learned and what is presented in a new situation, which may require rule abstraction. Generalization is typically assessed by training an organism on separate items or events that share one or several common features, and then testing whether the organism demonstrates knowledge about the relation between the items or events. Generalization occurs in the sensory domain (e.g. category learning), or involves more complex relations (transitive or associative inference). Generalization can stem from integrative encoding that occurs while experiencing events that partially overlap with previously encoded events, and purportedly depends on the hippocampus and the midbrain dopaminergic neurons (Shohamy and Wagner, 2008). Generalization of conditioned response is gauged in transfer tests which measure the response or selection rate for new exemplars of stimuli which resemble stimuli that have previously been conditioned. Results from such experiments can be illustrated by a stimulus generalization gradient which describes the rate at which the conditioned response declines as the stimulus is changed (along some discriminative dimension) from the CS+ to CS-. While in this sense transfer test is a method to verify generalization, in educational psychology, transfer, is the ability to apply learned knowledge to new situations. Transfer from the trained task to other even very similar tasks is generally an exception rather than the rule (Green and Bavelier, 2008). 
Learning is the process of acquiring new or modifying existing knowledge, skills, behaviors or preferences (Schacter, Gilbert, \& Wegner, 2010). Associative learning as well as declarative memory formation involve instance learning (encoding), followed by consolidation processes, resulting in a durable memory trace that can be accessed at some later time (retrieval). If the instance can be linked to existing knowledge, generalization may take place (at encoding or at a later stage of the memory formation process; Zeithamova, Schlichting, \& Preston, 2012), whereby the instance is bound to perceptually, contextually, or semantically similar items.

Motivation is a state of desire or energy to carry out a certain action, triggered by intrinsic or extrinsic factors (Pennartz et al. 2011). Motivation enhances performance and learning and is typically accompanied by increased arousal. In psychology, instrumental value is the object of extrinsic motivation, such as for example obtaining food or praise. Extrinsic motivation thus refers to doing something because it yields a tangible outcome (such as a rewarding gift) (Ryan \& Deci 2000). Very high extrinsic incentives may lead to "overmotivation" which undermines the beneficial effects of motivation and bears resemblance to loss-avoidance behavior (Chib et al., 2012; Mobbs et al., 2009), also related to activity in the ventral striatum. Intrinsic motivation refers to the drive to pursue goals that lead to a personal sense of competence and autonomy, providing inherent satisfaction and enjoyment (Ryan and Deci, 2000).

Reward (reinforcement) learning is an associative learning (conditioning) procedure in which the behavior is followed by a positive event. As a consequence, the behavior increases in strength, i.e. the rate and/or vigor of responding are increased (Bouton, 2007).

Rewards are considered as stimuli that positively reinforce behavior (Schultz, 2006) and that induce "wanting" - an energized state of motivation that affects performance and reward consumption (Berridge, 2003). Similarly to the "seeking disposition" (Alcaro and Panksepp, 2011), 
"wanting" involves the attribution of incentive salience. Incentive salience is proportionate to the value of an unconditional or reward-conditioned stimulus (cue) that captures attention, and triggers "wanting". Recognition of a reward results in reward anticipatory response. Reward processing involves the mesolimbic dopaminergic system (Flagel et al., 2011; see main text, section 2)

Schemas, in the memory domain, are considered as knowledge frameworks that capture regular patterns in the environment by abstracting information across experiences (Bartlett, 1932) and represent features common to multiple different events while discarding idiosyncratic details (Zeithamova et al., 2012). A schema is therefore an organized set of concepts that forms a unit of knowledge that can be invoked when experiencing a triggering object or event. Once activated, the schema will lead to a set of expectations about the object or event. A schema can assimilate a new memory that largely conforms to its expectations, potentially distorting details of the memory. Alternately, a schema can be modified to accommodate new memories that strongly contradict it (Lewis and Durrant, 2011).

\section{Box 2. Role of sleep in the consolidation and generalization of rewarded memories}

The role of sleep in memory consolidation as well as generalization is well established (Rasch and Born, 2013; Stickgold and Walker, 2013). In reward memory formation, dopamine, in addition to enabling late-LTP, contributes to offline consolidation processes that continue after a stimulusstimulus or stimulus-response learning and help better remember the learned association (Shohamy and Adcock, 2010; Wimber et al., 2011). Rodent research revealed that rewarded memories may be spontaneously reactivated during sleep, with a coordinated replay of neuronal activity in the hippocampus and in the ventral striatum during slow-wave sleep (Lansink, Goltstein, Lankelma, McNaughton, \& Pennartz, 2009; Pennartz et al., 2009). Moreover, generation of outcome predictions is proposed to rely on synaptic plasticity mechanisms boosted 
during slow-wave sleep (SWS) (Pennartz et al., 2009). Thus, reward does not only support memory consolidation by increasing neuroplasticity in the hippocampus via DA release at encoding, but also by potentiating selective replay during sleep after encoding (Singer \& Frank, 2009), recently confirmed also by optogenetic stimulation of mouse DA VTA-dorsal hippocampus pathway at encoding which enhanced spatial memory and increased reactivation of the stimulated neurons during sharp-wave ripples in post-encoding sleep (Mcnamara et al., 2014). In fact in humans, the mere expectation of future monetary reward has been shown to boost sleeprelated gains in memory for a trained finger-tapping sequence (procedural memory; Fischer \& Born, 2009; Oudiette, Antony, Creery, \& Paller, 2013; Wilhelm et al., 2011). Reward circuits may play a role not only in selective memory consolidation during sleep, but also in the generation of dreams. The Reward Activation Model (Perogamvros and Schwartz, 2012) proposes that phasic VTA dopamine signaling during REM sleep favors the off-line replay of recent memory traces during this sleep stage (the reoccurrence during off-line states of the firing patterns of hippocampal principal cells previously observed during active waking behavior). Accordingly, the activation of the mesolimbic-DA reward system during sleep would prioritize information with high emotional or motivational relevance for (re)processing during sleep and dreaming and may also be responsible for the generation of the motivational content of dreams.

Several studies suggest that sleep facilitates the formation of connections between related items, thus facilitating the emergence of logical inference, generalization of learned rules to a new situation, or insight about an unknown relationship in the previously learned information (Ellenbogen et al., 2007; Lewis and Durrant, 2011; Stickgold and Walker, 2013; Wagner et al., 2004). Sleep may also be important for the formation of 'mental schemas' - frameworks that capture regular patterns in occurrences by abstracting information across experiences (Bartlett, 1932)- which could emerge through the replay of overlapping events during slow wave sleep (Lewis and Durrant, 2011). In line with a computational model of memory by Kumaran \& McClelland (2012) which suggests the engagement of the prefrontal cortex in the formation of 
schemas, the mPFC in the rat (and presumably the vmPFC in humans) receives projections from the anterior hippocampus and accumulates information about the context of interrelated memories thus facilitating the retrieval of context-bound memories as well as the assimilation of new memories into pre-existing networks of knowledge (Preston and Eichenbaum, 2013; Figure 1). 


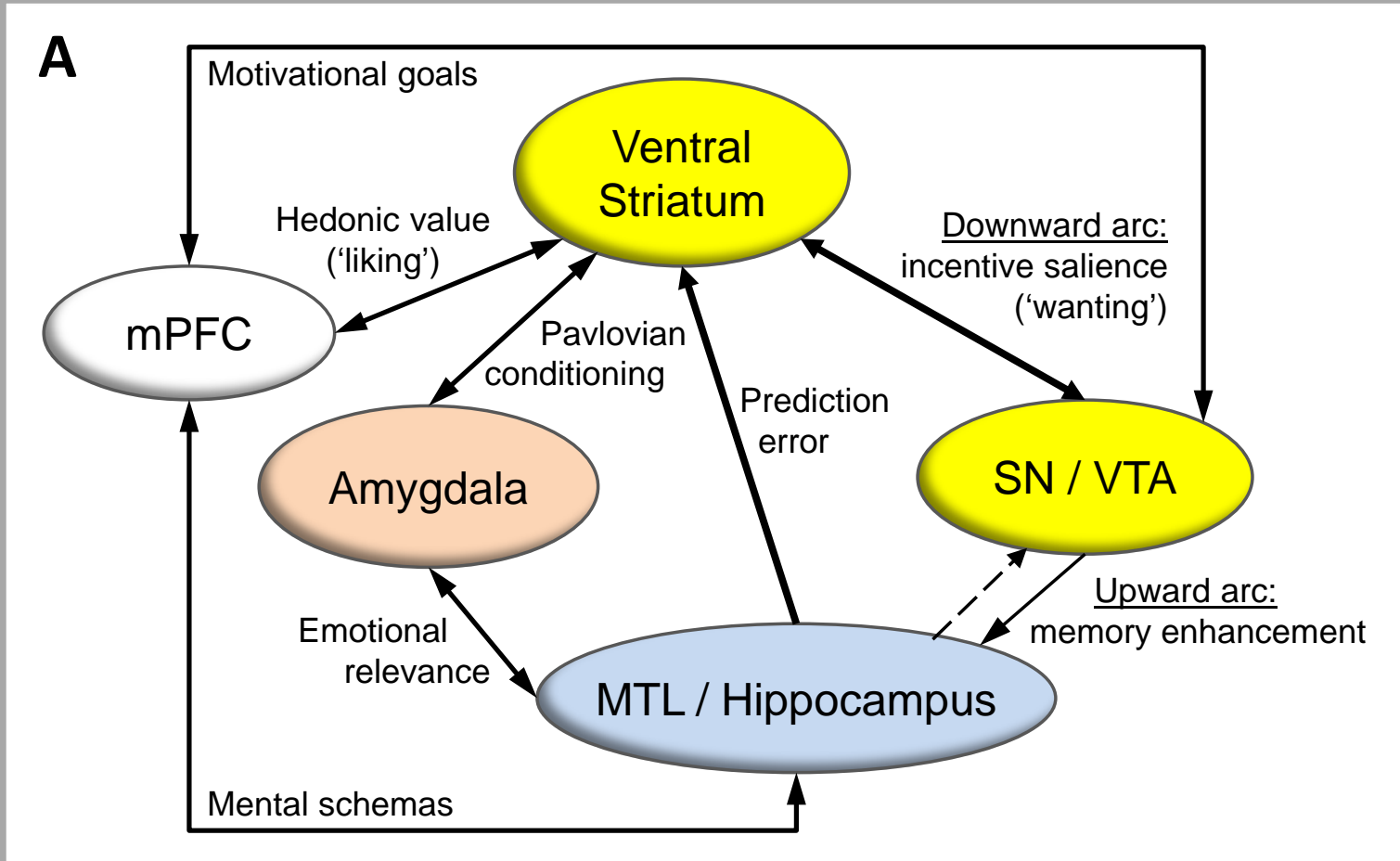

B

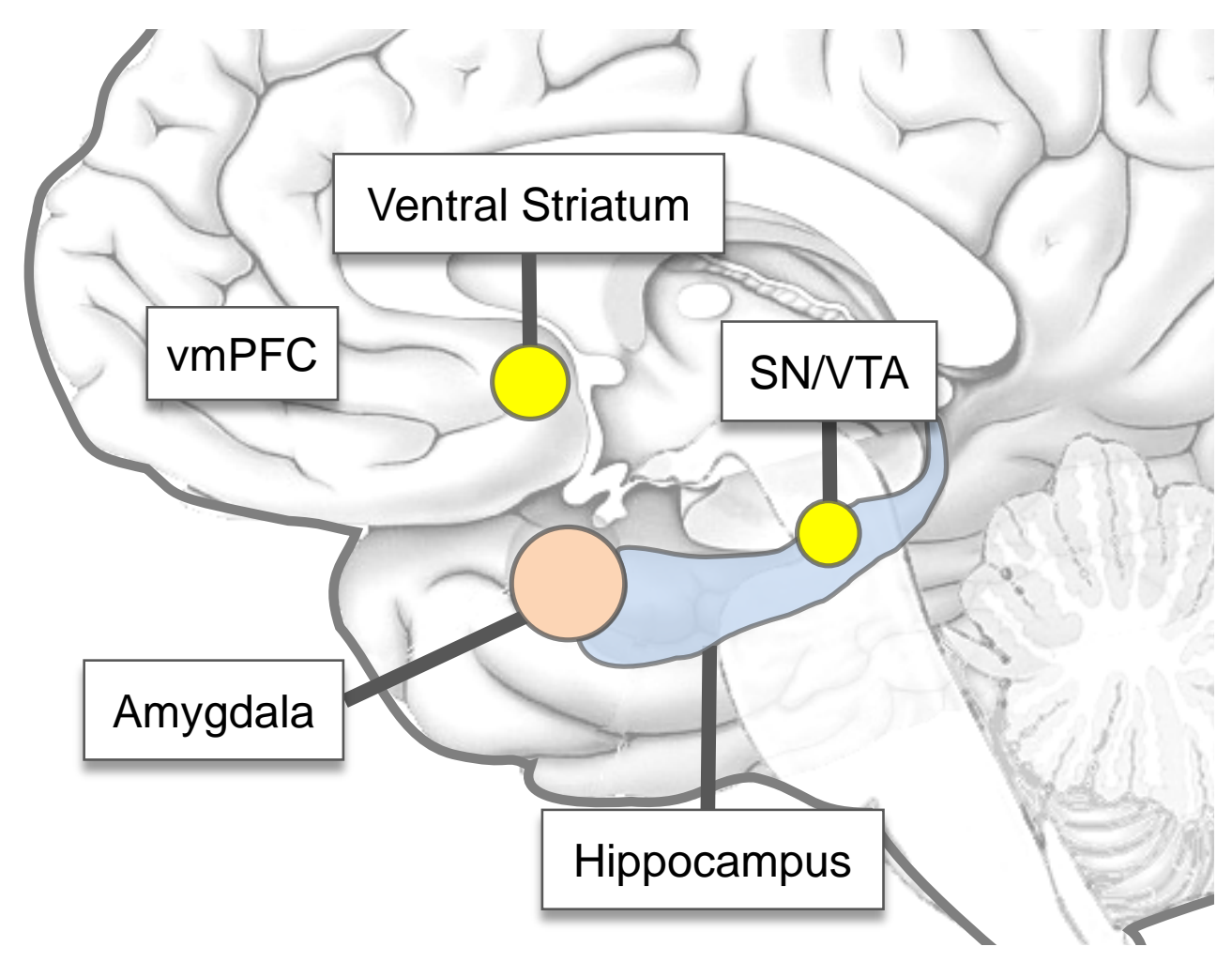

Figure 1 
A

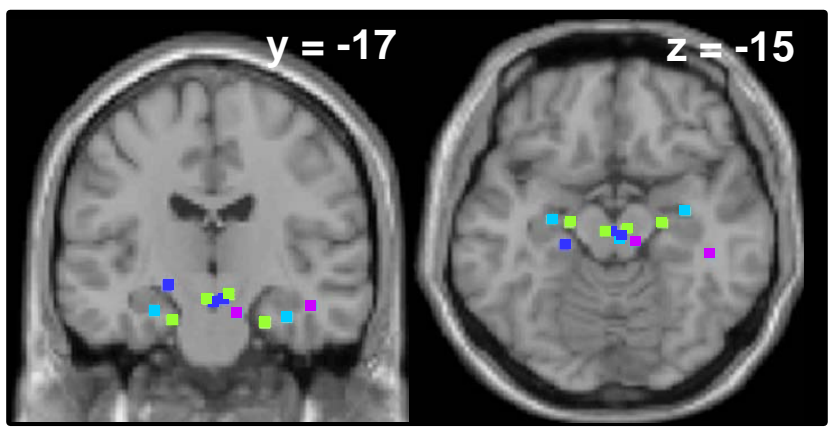

Recognized vs forgotten high-value cues (Adcock et al. 2006)

Recognized vs forgotten high-value cues (Wittmann et al., 2005)

Correlation of midbrain and hippocampus activation during a salient cue in a rewarded block (Murty \& Adcock, 2013)

Midbrain and hippocampal activity for late vs early learning correlates with the degree of generalization (Shohamy \& Wagner, 2008)
B

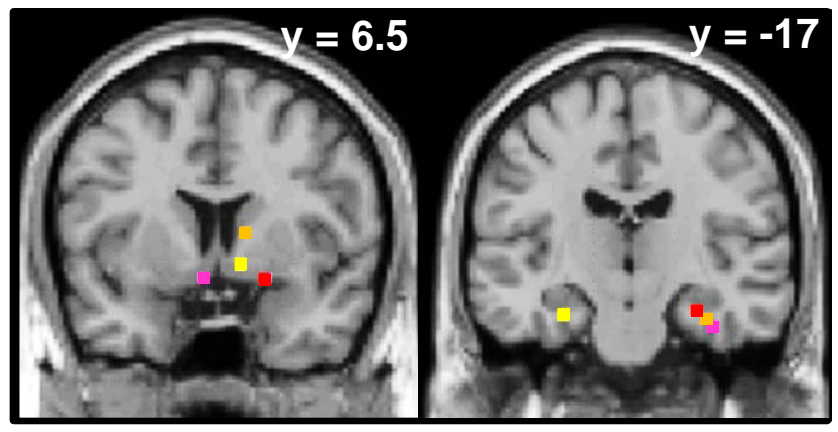

Pavlovian-to-Instrumental Transfer (Talmi et al., 2008)

Associative generalization on subsequent choice (Wimmer \& Shohamy, 2012)

Generalization of prediction error in outcomeprobability-association (Wimmer, Daw \& Shohamy, 2012)

Generalization of prediction error in perceptual similarity-generalization (Kahnt et al., 2012)

Figure 2 


\section{A: Similarity-based stimulus generalization}

2. Test: which grating predicts a reward?
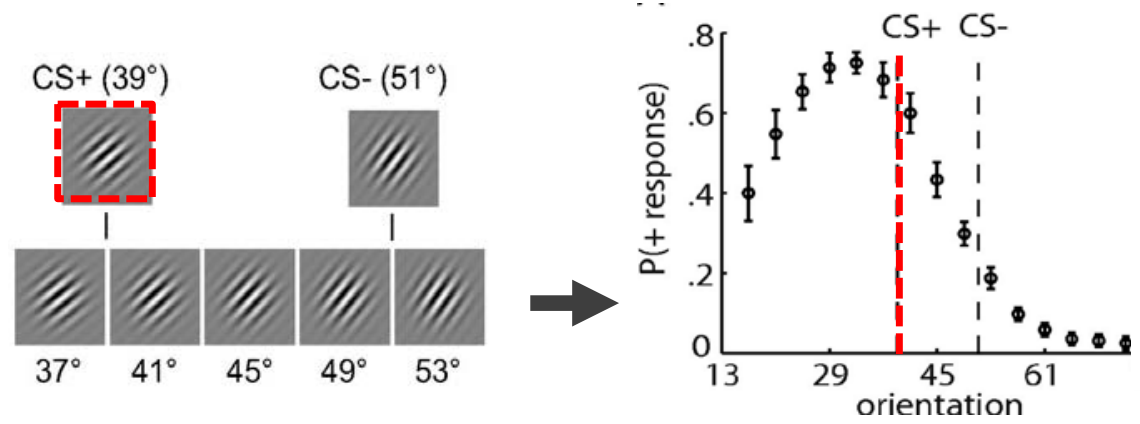

\section{B: Generalization via association or acquired equivalence}

1. Association learning

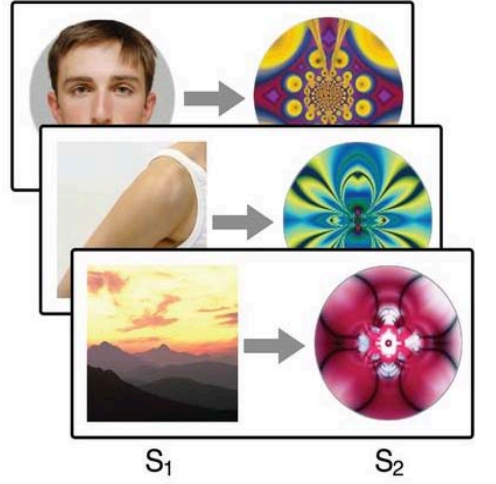

2. Reward conditioning
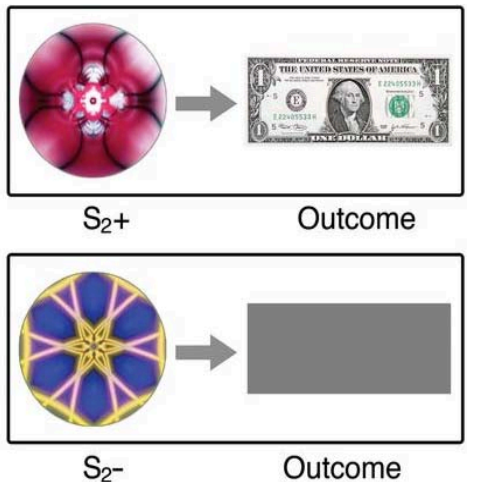

3. Test: which image predicts a reward?

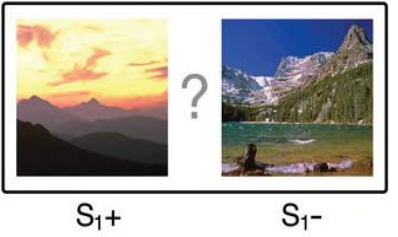

\section{C: Pavlovian-to-Instrumental Transfer (PIT)}

2. Instrumental learning

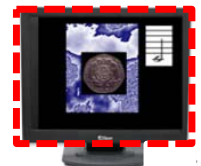

CS+
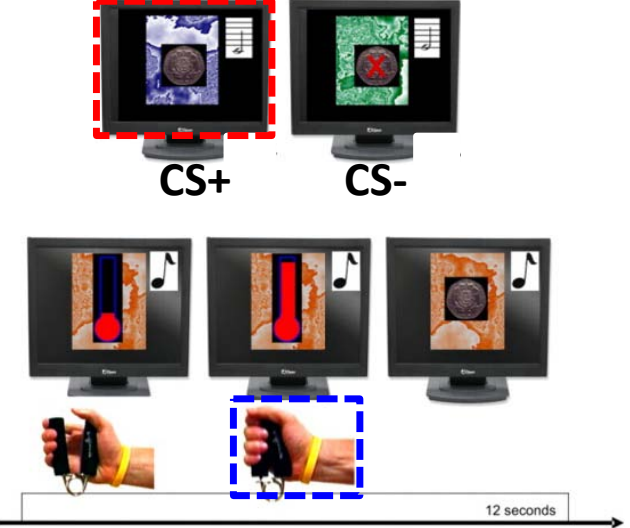

3. Pavlovian instrumental transfer

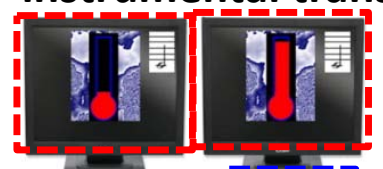

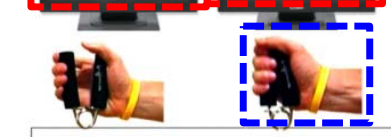

Figure 3 
A: Incidental learning during state of reward motivation

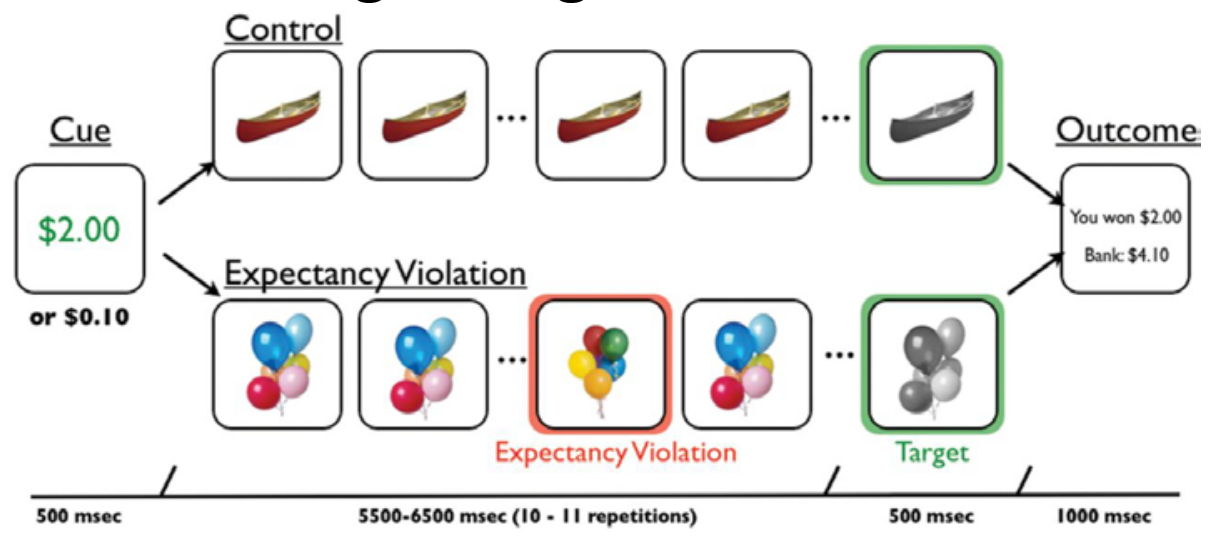

\section{B: Reward-related effects on memory integration}

1. Association learning with feedback

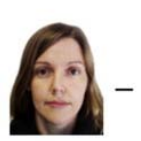

$\mathrm{F}_{1}$

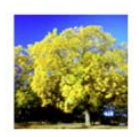

$\mathrm{S}_{1}$

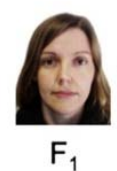

$F_{1}$

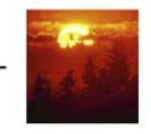

$\mathrm{S}_{2}$

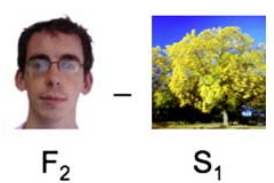

2. Test for generalization

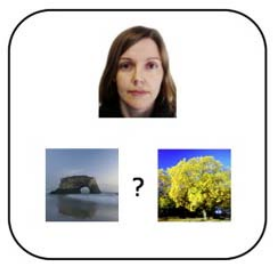

Trained

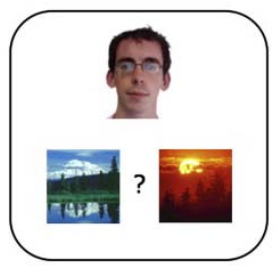

Generalization

C: Effect of reward in later memory transformation

1. Rewarded association learning

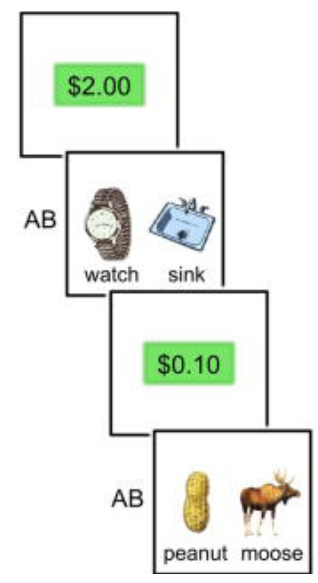

Encoding

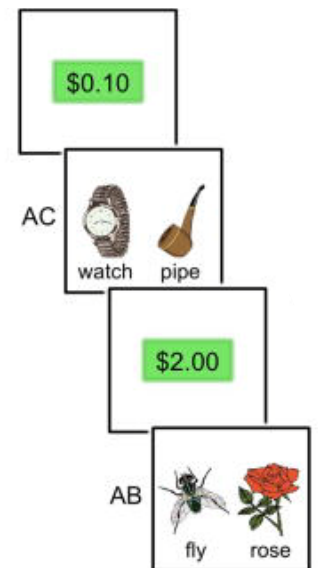

Interference
2. Test for generalization

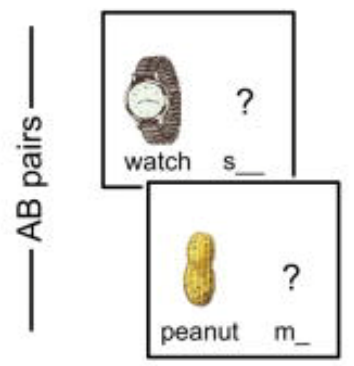

Figure 4 